41

\section{Chunking as a rational strategy for lossy data compression in visual working memory.}

${ }^{1}$ Department of Cognitive, Linguistic, and Psychological Sciences; Brown Institute

for Brain Science, Brown University 02912-1821

Matthew R. Nassar ${ }^{1}$, Julie C. Helmers ${ }^{1}$, and Michael J. Frank ${ }^{1}$

Corresponding Author:

Matthew R. Nassar

Department of Cognitive, Linguistic and Psychological Sciences

Brown University

Providence, RI 02912-1821

Phone: 607-316-4932

E-mail: matthew_nassar@brown.edu 


\section{Abstract:}

The nature of capacity limits for visual working memory has been the subject of an intense debate that has relied on models that assume items are encoded independently. Here we propose that instead, similar features are jointly encoded through a "chunking" process to optimize performance on visual working memory tasks. We show that such chunking can: 1) facilitate performance 8 improvements for abstract capacity-limited systems, 2) be optimized through reinforcement, 3) be implemented by center-surround dynamics, and 4) increase effective storage capacity at the expense of recall precision. Human performance on a variant of a canonical working memory task demonstrated performance advantages, precision detriments, inter-item dependencies, and trial-to-trial behavioral adjustments diagnostic of performance optimization through centersurround chunking. Models incorporating center-surround chunking provided a better quantitative description of human performance in our study as well as in a meta-analytic dataset, and apparent differences in working memory capacity across individuals were attributable to individual differences in the implementation of chunking. Our results reveal a normative rationale for centersurround connectivity in working memory circuitry, call for re-evaluation of memory performance differences that have previously been attributed to differences in capacity, and support a more nuanced view of visual working memory capacity limitations: strategic tradeoff between storage capacity and memory precision through chunking contribute to flexible capacity limitations that include both discrete and continuous aspects.

43 Keywords: Visual working memory; Data compression; Information theory;

44 Reinforcement learning; Computational modeling; Recurrent neural networks 


\section{Introduction:}

People are limited in their capacity to retain visual information in short-term

4 memory; however, the exact nature of this limitation is hotly debated (Luck \& Vogel,

5 2013; Ma, Husain, \& Bays, 2014; Wei, Wang, \& Wang, 2012). Competing theories

6 have stipulated that capacity is constrained by either a discrete item limit (e.g., a

7 fixed number of "slots") or by the distribution of a flexible "resource" across

8 relevant visual information (Bays \& Husain, 2008; Wei et al., 2012; Zhang \& Luck,

9 2008). In their simplest form, these competing theories are both philosophically

10 distinct and statistically identifiable, but experimental evidence has been mixed,

11 with some studies favoring each theory and the best-fitting computational models

12 incorporating elements of each (Almeida, Barbosa, \& Compte, 2015; Bays \& Husain,

13 2008; Bays, Catalao, \& Husain, 2009; Cowan \& Rouder, 2009; Chris Donkin, Tran, \&

14 Nosofsky, 2013a; Christopher Donkin, Nosofsky, Gold, \& Shiffrin, 2013b; Rouder et

15 al., 2008; van den Berg, Awh, \& Ma, 2014; van den Berg, Shin, Chou, George, \& Ma,

16 2012; Zhang \& Luck, 2008; 2009; 2011). Experimental support for both theories has

17 emerged from delayed report working memory tasks, in which subjects are asked to

18 make a delayed report about a feature (e.g. color) of a single item that was briefly

19 presented as part of a multi-item stimulus display (Bays \& Husain, 2008; Wilken \&

20 Ma, 2004; Zhang \& Luck, 2011). In particular, as the number of items to be retained

21 increases, visual working memory reports tend to become less precise, as predicted

22 by resource models, and more likely to reflect guessing, as predicted by slots models 
1 (Fougnie, Suchow, \& Alvarez, 2012; Luck \& Vogel, 2013; Ma et al., 2014; van den

2 Berg et al., 2012; 2014).

3 While the competing classes of visual working memory models have evolved

4 substantially over the past decade, the mathematical formalizations of each have

5 relied on assumptions about what is, and should be, stored in working memory.

6 Thus, an open question with potentially broad implications is what should and do

7 people store in memory during performance of the standard delayed recall tasks,

8 and how do deviations from the standard assumptions affect our understanding of

9 memory capacity? To this end, recent work has highlighted the ability of people to

10 optimize memory encoding and decoding processes by pooling information across

11 memoranda to enhance performance under different regimes (Brady \& Alvarez,

12 2011; 2015; Brady, Konkle, \& Alvarez, 2009; Lew \& Vul, 2015; Orhan \& Jacobs,

13 2013; Sims, Jacobs, \& Knill, 2012; Wei et al., 2012). Specifically, people can integrate

14 prior information to improve memory report precision (Bays et al., 2009; Brady \&

15 Alvarez, 2011) and, when stimuli are redundant, lossless compression strategies can

16 be used to efficiently encode them (Bays et al., 2009; Brady et al., 2009; Zhang \&

17 Luck, 2008). These strategies can improve memory performance, but only to the

18 extent to which features of upcoming memoranda are predicted by previously

19 observed stimulus statistics. Since memoranda in standard working memory tasks

20 are unpredictable and randomly distributed by design, such strategies cannot

21 improve and may actually impede performance in standard tasks (Bays et al., 2009;

22 Orhan \& Jacobs, 2014; Zhang \& Luck, 2008). However, while memoranda in these

23 tasks are not compressible in the "lossless" sense, it is still possible that people 
1 might employ more fast and frugal techniques to reduce memory storage

2 requirements at a small but acceptable cost to task performance.

3 Here we explore this possibility and show that people should, could, and do

4 implement a lossy form of data compression that sacrifices information about subtle

5 differences in the feature values of memoranda in order to improve overall task

6 performance. We do so using an inter-related set of computational models across

7 different levels of analysis, such that we can constrain our understanding of the

8 compression algorithm using both computational notions of how information

9 should be compressed and mechanistic notions of how biological circuits could

10 implement this compression. We probe our own and published empirical data to

11 test key predictions of these models. This study thus involves four related

12 components:

13

14 1. Normative and behavioral analysis. We begin with an information-theoretic

15 analysis of how features of memoranda should be stored to maximize task

16 performance in an abstract memory-limited system. We show that that under

17 high memory load conditions, it is advantageous to jointly encode (chunk) a

18 blended representation of similar features and only separately encode

19 (partition) features if they are sufficiently dissimilar. This strategy can be

20 effectively implemented by setting a criterion for partitioning features based

21 on dissimilarity, where the appropriate criterion can be learned based on

22 trial feedback (binary reward) and increases with memory load. We show

23 that human subject behavior in a delayed report working memory task 
1 conforms to predictions from this form of adaptive chunking and

2 optimization thereof via reward feedback.

2. Mechanistic implementations of chunking in a biophysical network model.

Given that behavioral data accorded with predictions from the normative model, we next examined how such selective chunking could be implemented in a biophysical memory system. Established recurrent neural network models have shown that perceptually similar memoranda can be merged together via attractor "bump collisions" (Wei et al., 2012). However, these simulations showed that such an architecture leads to indiscriminate chunking and - due to lateral inhibition - increased forgetting of nonchunked items, leading to poor performance (Wei et al., 2012). We show that this issue can be remedied by adopting a more biologically motivated centersurround inhibition connectivity that effectively partitions dissimilar color

15 representations; however, it does so at the cost of inter-item repulsions, 16 which reduce the effective precision of memory reports (Almeida et al., 17 2015).

3. Algorithmic model of center-surround dynamics. To systematically explore the impact of center-surround mediated chunking at the behavioral level, we created a parsimonious (i.e., minimal parameter) model that incorporates the key features necessary for effective chunking afforded by the biophysical model without explicitly modeling the temporal dynamics or biophysical 


\section{Results:}

21 Visual working memory capacity is typically measured using either delayed report

22 or change detection tasks (Bays \& Husain, 2008; Wilken \& Ma, 2004; Zhang \& Luck,

23 2011). Here we focus on the former, as they have provided nuanced information

properties. We show that center-surround dynamics facilitate improved memory recall at the expense of precision, and capture previously unexplained qualitative patterns of bias and precision in human memory reports across stimulus arrays.

4. Quantitative model fitting. Finally, we fit behavioral data from individual subjects to show that chunking and center-surround dynamics improve fit relative to state-of-the-art models, and can account for considerable performance differences across subjects, with better-performing subjects best fit by models with more inclusive chunking policies. We validate these findings in a meta-analytic dataset to show that chunking improves quantitative model fits across tasks, offers an alternative explanation to changes in precision with set size, and accounts for individual differences in working memory task performance under conditions of high memory load. 
1 about the shape of memory distributions and have formed the basis for competing

2 models of capacity limitation (Fougnie et al., 2012; Luck \& Vogel, 2013; Ma et al.,

3 2014; van den Berg et al., 2012).

4

5 Specifically, we consider a delayed report color reproduction task that requires

6 storage of color and orientation information (figure 1). Each trial consists of three

7 core stages: stimulus presentation, delay, and probe. During stimulus presentation,

8 five oriented colored bars are displayed simultaneously. During the subsequent

9 delay, the screen is blanked, requiring short-term storage of color and orientation

10 information. During the probe stage, a single oriented bar is displayed (in gray) and

11 the participant is required to reproduce the color that had been associated with that

12 orientation in the preceding stimulus array.

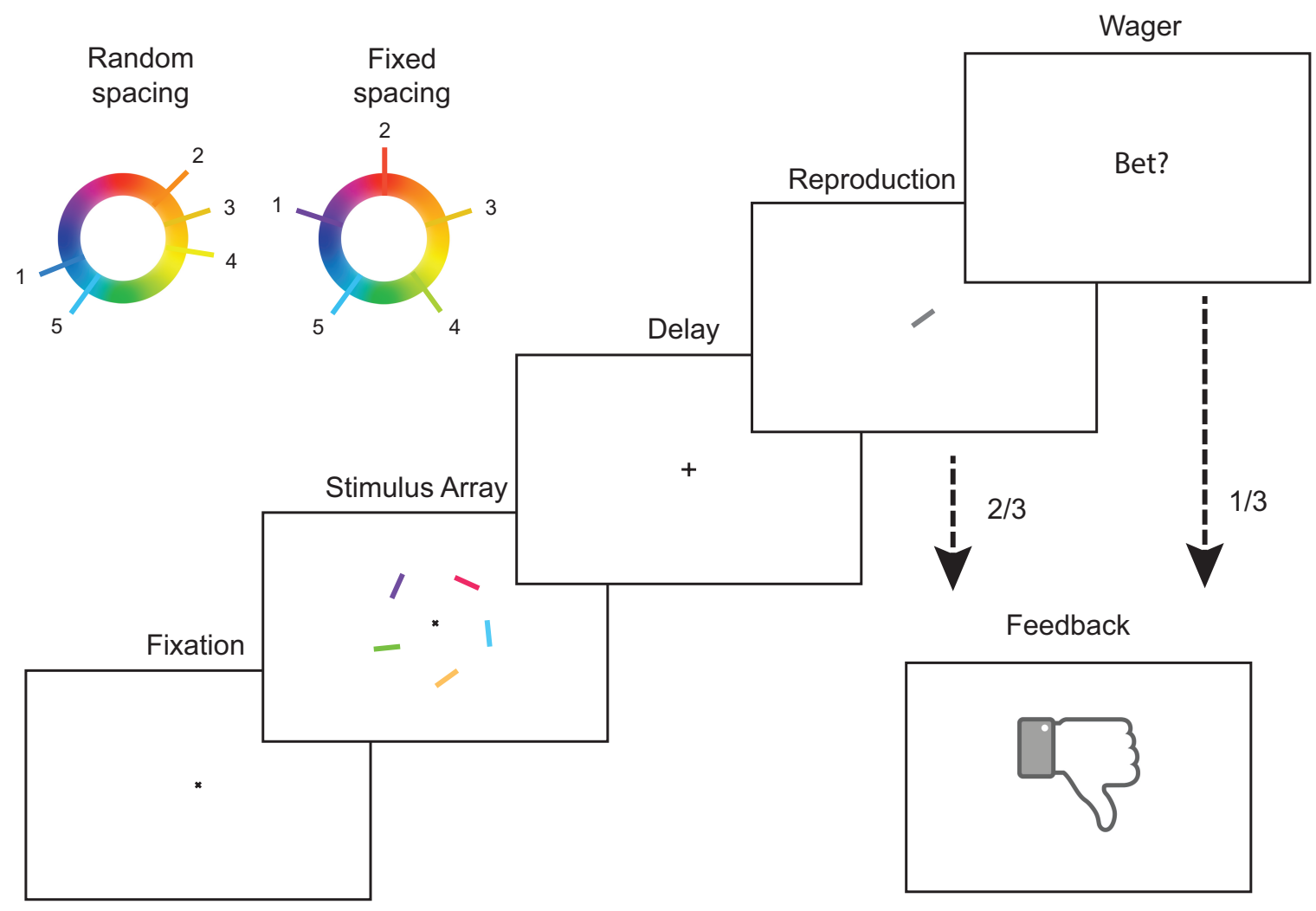


1 Figure 1: Delayed report color reproduction task. Each trial begins with central fixation for 500

$2 \mathrm{~ms}$, followed by stimulus presentation for $200 \mathrm{~ms}$. Stimuli consist of five colored and oriented bars

3 evenly distributed around a circle subtending 4 degrees of visual angle and centered on the point of

4 fixation. Stimulus presentation is followed by a $900 \mathrm{~ms}$ delay, after which a single oriented bar is

5 displayed centrally. The subject is required to report the color associated with the bar with the

6 probed orientation in the previous stimulus array. After confirming the report, the subject receives

7 feedback dependent on whether the absolute magnitude of the reproduction error was greater or

8 less than a fixed threshold. Stimulus colors on any given trial are selected either: 1) randomly and

9 independently as is standard in such tasks (random spacing; upper left) or 2) ensuring uniform

10 spacing on the color wheel so as to minimize within-array color similarity (fixed spacing).

12 Information theoretic analysis of what should be stored in working memory.

14 To first understand whether, in principle, information encoding could be optimized

15 in this task, we developed a limited-capacity system for memory storage in which

16 colors and orientations are represented with binary words (figure 2). We build on

17 the now classical work of George Miller by conceptualizing working memory

18 capacity limitation in terms of a strict limit on information storage, in bits (G. A.

19 Miller, 1956). The precision with which a color is stored depends on the number of

20 binary digits (bits) used to represent that color: a single bit can be used to specify a

21 half of the color wheel, a second bit can be added to specify a quarter of the color

22 wheel, and so on (figure 2A,). Capacity limitations within such a system can be easily

23 implemented as a fixed limit on the number of bits stored during the delay period.

24 These bits can be used to represent the individual colors in the target array, by, for

25 example, dividing them evenly among the available targets (figure 2B). 
1 Alternatively, multiple similar colors could be jointly represented with a single

2 binary word that is then linked to multiple orientations (figure 2C). An intuitive

3 advantage of the second encoding strategy is that reducing the number of binary

4 color words increases the number of bits available to represent each word,

5 potentially offsetting the biased encoding of the chunked items by better

6 representing each encoded color.

A

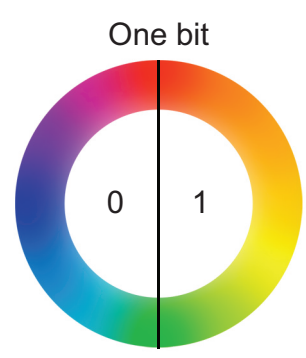

Two bits

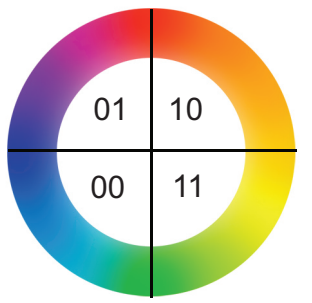

Three bits

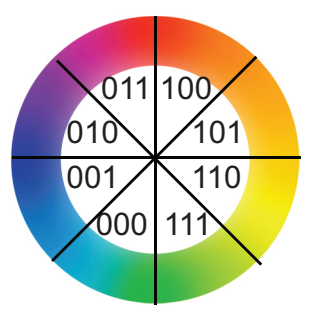

Stimulus:

B

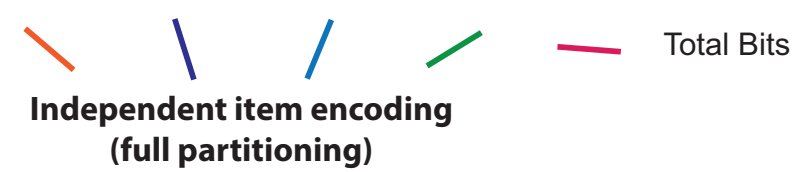

(full partitioning)

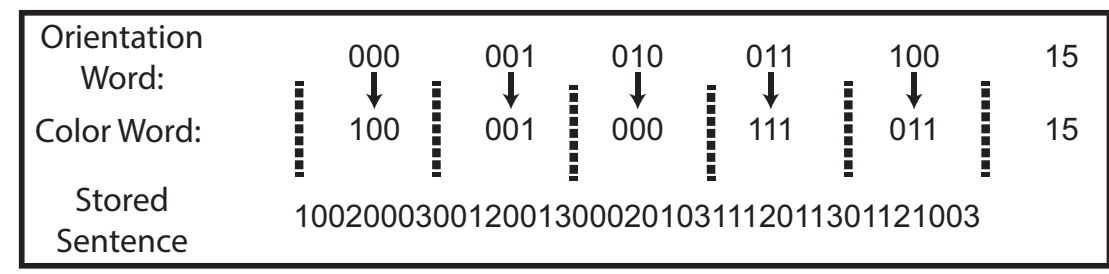

\section{C $\quad \begin{gathered}\text { Chunking } \\ \text { (selective partitioning) }\end{gathered}$}

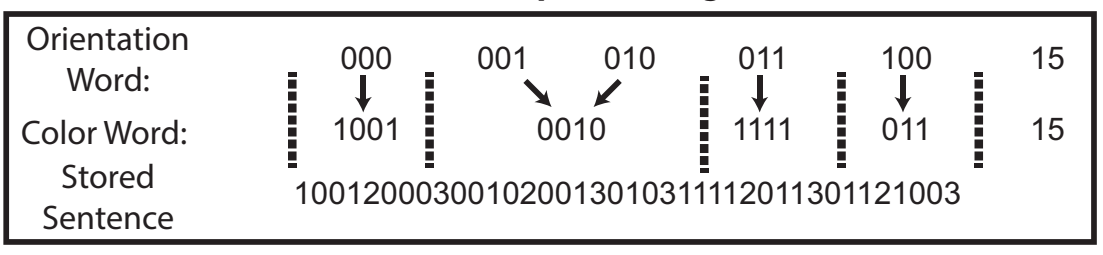

D

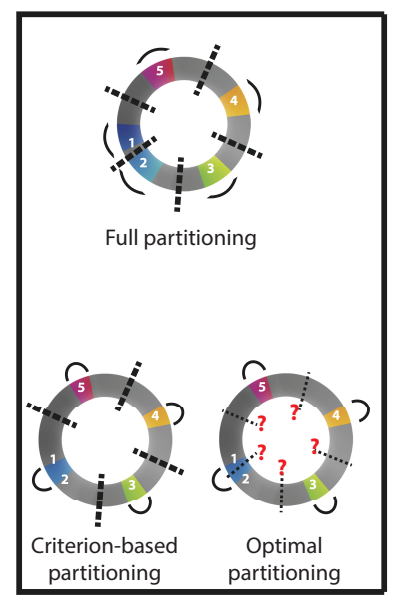

10 Figure 2: Binary encoding model of visual working memory. In order to formalize capacity

11 limitations, it is useful to consider an abstract model of working memory that stores features in

12 binary words. A: Each color can be described by a binary word of fixed length, where the number of 
1 digits in the word determines the storage precision. B \& C: Stimulus arrays can be stored by linking

2 ordered pairs of color and orientation words. Capacity limitations are modeled by a fixed limit on the

3 length of the resulting "sentence" comprised of color and orientation words separated by word

4 termination symbols (2/3 for color/orientation words, respectively). B: One strategy for storing

5 ordered pairs involves alternating sequences of color and orientation words, such that each color is

6 "partitioned" from all other colors (dotted lines separating color representations) and linked to a

7 single orientation. C: Another strategy for storage would be to link two or more orientations to a

8 single color by removing a partition (chunking). This reduces the number of colors that need to be

9 stored, and thus increases the number of bits allotted to each color. D: Full partitioning (top) involves

10 placing a partition between each set of colors such that each color is represented independently.

11 Criterion-based partitioning sets a partition between each set of colors that are separated by a

12 greater distance than the partitioning criterion. Optimal partitioning examines all partitioning

13 patterns for a given stimulus array and selects the partitioning pattern that would achieve the lowest

14 theoretical error magnitude. Colors/Arcs in each model reflect stored representations of a

15 particular stimulus array (actual stimuli labeled with numbers) and thick/thin lines indicate

16 actual/potential partitions. Note that in this case, actual partitions selected by optimal

17 partitioning do not differ from those selected by the criterion-based partitioning model.

21 To test this potential advantage quantitatively, we examined task performance of

22 three models that differ in how they "chunk" feature information. The standard

23 model employs independent item encoding, through which each color is partitioned,

24 or represented separately, from all other stored colors (full partitioning; figure

25 2B\&D). We also consider a fully optimal model that considers all possible

26 partitioning patterns and stores information using the combination of partitioning 
1 and chunking (figure 2C\&D) that would lead to the best theoretical performance for

2 each specific stimulus array, given the possibility that any item could be probed

3 (optimal partitioning). Although determining the optimal partitioning pattern for

4 each array is computationally expensive, it is well approximated by a simple and

5 efficient heuristic, using a single criterion for partitioning, according to the

6 separation of two targets in color space (criterion-based partitioning; fig 2D). For

7 this model, chunking was parameterized by a "partitioning criterion" that defines

8 the minimum distance between two colors required for independent

9 representation. If the distance between two colors is smaller than the partitioning

10 criterion, the colors are represented as a single "chunk". Thus, a partitioning

11 criterion of zero indicates that all items are represented independently, whereas a

12 partitioning criterion of $\pi$ indicates that all item colors will be chunked together (i.e.

13 represented by a single binary word). Performance of all models was assessed for

14 delayed recall tasks ranging from easy (2 items) to difficult (8 items) and using both

15 continuous and discrete assumptions regarding the distribution of item information.

17 Performance of the criterion-based model depended on partitioning criterion as a

18 function of task difficulty (figure 3). For easier tasks with few items to encode, the

19 model's memory buffer was large enough to store each item independently with a

20 reasonable number of bits, such that increasing the partitioning criterion beyond

21 zero was detrimental to task performance (two targets; figure 3a [dark line]).

22 However, for harder tasks, in which storing each item with high precision was not

23 possible due to limited buffer size, performance was best for moderate partitioning 
1 criterions that allow for joint representation of similar, but not dissimilar, colors

2 (eight targets; figure 3a [light line]). To better understand how chunking interacts

3 with set size to affect performance, we compared the performance of the best

4 criterion-based partitioning model to that of a full partitioning model across

5 different task difficulties. Across task difficulties, there was a monotonic

6 relationship between the number of targets and the performance advantage of both

7 fully optimal and criterion-based chunking models over full partitioning (figure 3B;

8 compare orange and green lines). Furthermore, the performance of the best

9 criterion-based partitioning was nearly identical to that of the optimal partitioning

10 model (figure 3B; compare green and yellow lines). Notably, the number of colors

11 stored by criterion-based and optimal partitioning models saturated around four

12 with increasing set size, highlighting that the improved performance of these

13 models comes from chunking similar colors into a single representation that is

14 linked to multiple orientations. Moreover, this result suggests that even though it is

15 possible in these models to store more items independently with less precision, it is

16 more advantageous to restrict the number of stored representations during

17 standard delayed recall tasks (figure 3C).

19 Set-size dependent performance advantages of chunking were also relatively

20 insensitive to modeling assumptions regarding the nature of storage constraints.

21 While the results described above were generated under the assumption of a

22 divisible resource framework (figure 3A-C), comparable results were attained when

23 model behavior was simulated using binary words that roughly correspond to the 
1 slots + averaging framework (figure 3D-F). Thus, the performance advantages

2 offered by criterion-based chunking are robust to the nature of the actual capacity

3 limitation.

5 Adaptation of partitioning criterion via reinforcement learning

7 The advantages of chunking discussed above were presented for the best

8 partitioning criterion, which differed as a function of task demands, begging the

9 question of how a human subject would know to use this criterion. We thus

10 examined whether the partitioning criterion could be optimized on a trial-to-trial

11 basis via reinforcement learning to improve performance by allowing the

12 partitioning criterion to be adjusted on each trial according to the chunking (total

13 number of chunks) and reward feedback (thresholded binary feedback) from the

14 previous trial (see Methods). The resulting model increased the partitioning

15 criterion, and thus chunking, after a trial in which it chunked colors and achieved

16 better-than-average performance. This led partitioning criterions to increase

17 rapidly towards a load-dependent asymptote (figure 3G). Trial-to-trial increases in

18 the partitioning criterion corresponded to rapid improvements in overall task

19 performance, as measured by average absolute error (figure $3 \mathrm{H}$ ). These

20 improvements in task performance were concomitant with reductions in the total

21 number of color chunks that the model attempted to store (figure 3I). Thus,

22 chunking can be optimized across conditions through reinforcement learning to 
1 improve performance and reduce effective storage requirements in higher memory

2 load contexts.

3

$4 \quad$ Given the performance advantages offered by criterion-based chunking and the

5 efficiency with which it could be learned and implemented, we next sought to

6 identify diagnostic predictions made by the model. One key difference between the

7 criterion-based and full partitioning models is that the performance of the former

8 depended heavily on the specific distribution of the colors within each stimulus

9 array. If the colors were randomly and independently sampled, which is the

10 standard method in such tasks, chunking offered large advantages. In contrast, the

11 advantages of chunking were considerably smaller when colors were uniformly

12 distributed in color space to maximize color separation (fixed spacing; figure 1

13 inset; compare the solid and dashed lines in figure 3E-F). It is noteworthy that the

14 prediction of performance decrements under fixed spacing conditions is completely

15 opposite to that made by a prominent active maintenance model of working

16 memory, a point that we address in detail with a biologically motivated model of

17 chunking in a later section (Wei et al., 2012). 
A

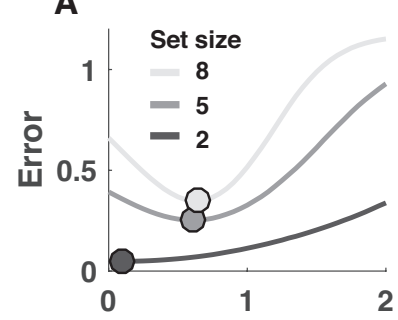

D

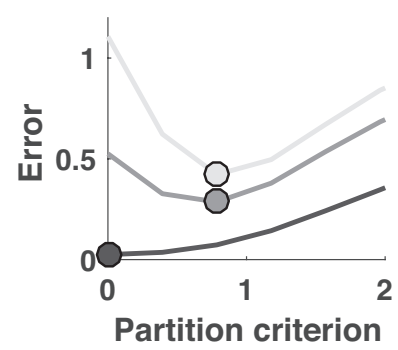

G

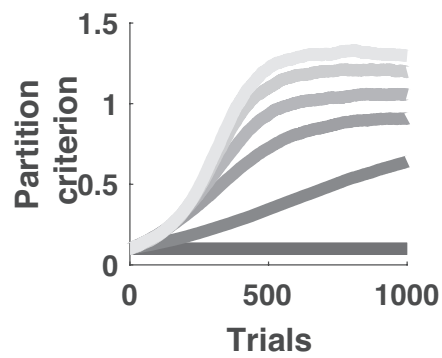

$\mathbf{J}$

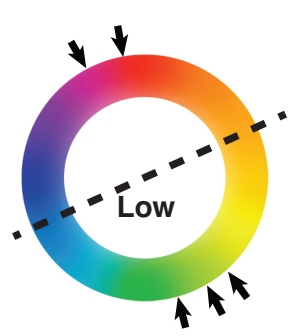

Within cluster variance

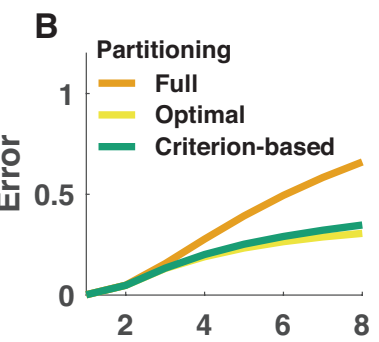

E

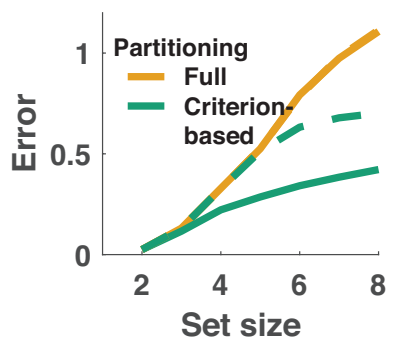

H

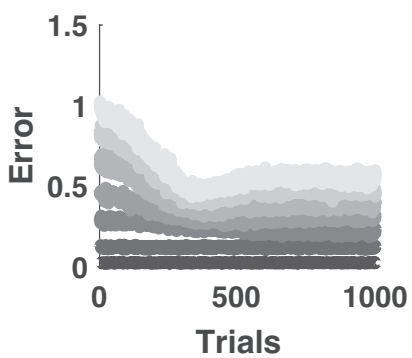

K

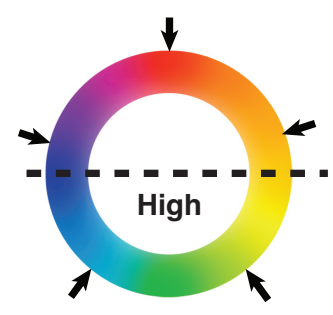

ce

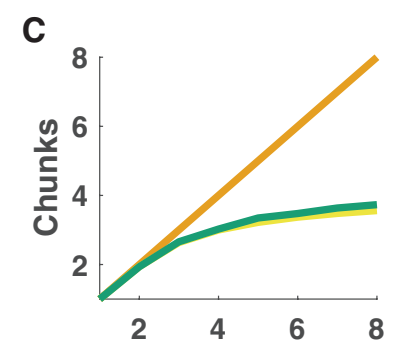

F

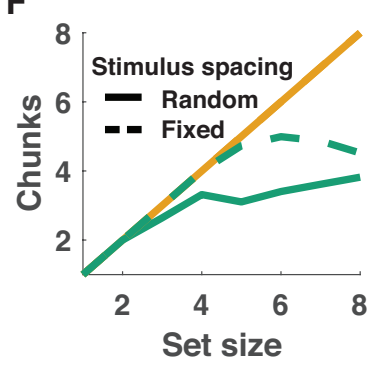

I
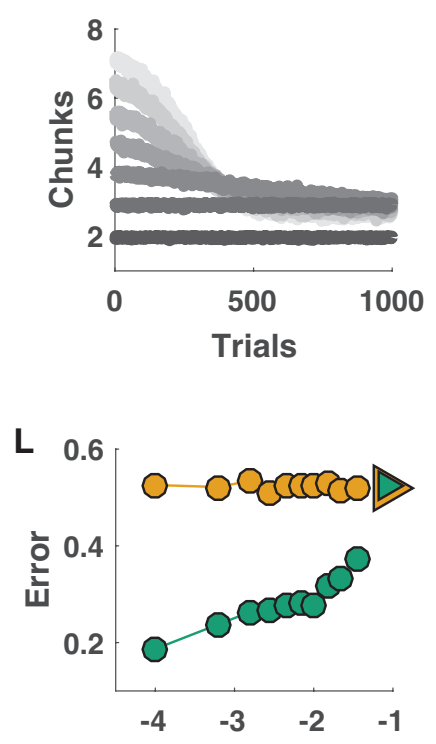

Within cluster variance

3 Figure 3: Chunking improves memory performance and can be achieved through trial-to-trial

4 adjustments of partitioning criterion. A-C: Criterion-based chunking confers memory 
1 performance advantages and reduces feature storage requirements under resource assumptions. A:

2 Mean absolute error (ordinate) for theoretical performance of a binary encoding model on delayed

3 report tasks of varying set size (grayscale) across all possible partitioning criterions (abscissa; $0=$ all

4 colors stored independently). B: Model error (ordinate) increases as a function of set size (abscissa)

5 for three partitioning strategies: 1) fully partitioned (model always stores all targets independently),

6 2) optimal partitioning (model considers all possible partitions for each stimulus array and uses the

7 best), 3) criterion-based partitioning (chunking and partitioning is determined by best criterion from

8 A). Error increases more shallowly for optimal and criterion-based partitioning strategies that

9 employ strategic chunking. C: Total number of chunks requiring storage (ordinate) increases as a

10 function of set size (abscissa) for all three models, but saturates near 4 items for optimal and

11 criterion-based chunking models. D-F: Performance advantages of criterion-based chunking hold for

12 binary word storage, analogous to "slots + averaging." D-F are analogous to A-C except that panels E

13 and F show model performance separately for randomly spaced and fixed spaced stimulus arrays

14 (solid and dotted lines, respectively) and do not include an "optimal partitioning" model, as

15 computing it would be computationally inefficient under this framework. G-I: Appropriate

16 partitioning criterions can be learned through reinforcement. G: Adjusting the partitioning criterion

17 through reinforcement learning (see Methods) leads simulated criterions (ordinate) to increase over

18 trials (abscissa) in a manner that scales with set size (grayscale; $2=$ darkest, $8=$ lightest).

19 Adjustments in criterion lead to reduced errors $\mathbf{( H )}$ and decrease the "chunks" that require storage

20 (I). J-L: Chunking selectively benefits performance on trials in which colors are most tightly

21 clustered. Within-cluster variance provides a measure of feature clustering within a stimulus array,

22 with low values indicating more clustering $(\mathbf{J})$ and high values indicating less clustering (K).

23 Performance of the best chunking model, but not the non-chunking model, depends on the clustering

24 of individual stimulus arrays, as assessed through within-cluster variance. Mean absolute error is

25 plotted for stimulus arrays grouped in bins of within-cluster variance for criterion-based chunking

26 (green) and fully partitioned (orange) models. Triangles reflect the same values computed for fixed

27 spacing trials, in which stimulus features were minimally clustered (as depicted in $\mathbf{K}$ ). 
2 To better characterize the statistical properties of the specific arrays that enabled

3 better performance in the chunking models, we computed the within-cluster

4 variance (WCV) as a metric of how efficiently each stimulus array could be chunked

5 (see Methods). Target arrays with tightly clustered colors have low WCV, whereas

6 target arrays with distantly spaced colors have high WCV (figure 3J\&K). The

7 performance of chunking models depended monotonically on WCV, with the

8 smallest errors achieved on low WCV target arrays (figure 3L), supporting the

9 notion that chunking advantages are achieved by more efficient representation of

10 similar colors through joint encoding.

11

12 Taken together, the results from the binary encoding model suggest that 1)

13 selectively chunking similar feature values can improve performance in working

14 memory tasks, 2) performance improvements from selective chunking increase with

15 target number and are mitigated by uniformly spacing feature values, 3)

16 performance of chunking models depends monotonically on WCV, and 4) chunking

17 behavior can be learned through reinforcement of successful chunking behaviors. In

18 summary, the binary encoding model clarifies why, and under what conditions,

19 chunking could benefit working memory performance in standard tasks. In the next

20 section, we test whether performance of human subjects in a delayed recall task

21 conforms to the predictions of the criterion-based chunking model.

23 People are more accurate and confident when remembering clustered stimulus arrays. 
2 To directly test key predictions made by the binary encoding model, we collected

3 behavioral data from human participants in the task described in figure 2. The

4 critical manipulation in the task is that the colors in some trials were uniformly

5 spaced on the color wheel (fixed spacing) whereas the colors in interleaved trials

6 were randomly and independently sampled from the color wheel (random spacing).

7 This manipulation allowed us to test the prediction of the binary coding model that

8 more clustered stimulus arrays lead to better performance (figures 3E\&I). To

9 examine the potential for adaptive learning of chunking, we provided reward

10 feedback to subjects on each trial (by comparing error magnitude to a fixed

11 threshold, either $\pi / 3$ or $\pi / 8$ in separate groups). The task also required subjects to

12 wager about the accuracy of their choices (post-decision) on one third of trials.

13 These task features allowed us to test the prediction that chunking behaviors are

14 adjusted from trial-by-trial through reinforcement learning (see figure 3G\&H) and

15 to determine whether participants were aware of any performance bonuses

16 attributable to chunking.

17

18 In accordance with behavioral optimization through selective chunking, participants

19 were more accurate and confident when presented with randomly spaced stimuli.

20 Subject accuracy, as assessed using the same error threshold procedure used to

21 determine feedback, was greater on random spacing trials than on fixed spacing

22 trials (figure S1A; $\mathrm{t}=4.4, \mathrm{p}<10 \mathrm{e}-4$ ). This accuracy improvement was more

23 prevalent for subjects that experienced a liberal accuracy criterion (low precision, 
1 absolute error $<\pi / 3$ ) than for those that experienced a conservative accuracy

2 criterion (high precision, absolute error $<\pi / 8$; two sample t-test for group

3 difference: $\mathrm{t}=-2.5, \mathrm{p}=0.02$, Bayes Factor $=4.8$ ) suggesting the improvement may

4 have been more pronounced for moderate errors that were interpreted differently

5 across the groups depending on the accuracy criterion (error $<\pi / 3$ \& error $>\pi / 8$ ).

6 Participants also wagered more frequently on random spacing than fixed spacing

7 trials, suggesting that they were cognizant of the conferred performance advantage

8 (figure $\mathrm{S} 1 \mathrm{~B} ; \mathrm{t}=3.1, \mathrm{p}=0.003$ ). Subjects tended to gauge their wagering behavior

9 reasonably well, with subjects who achieved higher levels of accuracy also betting

10 more frequently (figure S1C; Pearson's rho =0.68, p <10e-6). Furthermore,

11 individual differences in the adjustment of wagering as a function of color spacing

12 configuration correlated with the change in accuracy that subjects experienced

13 across the configurations (figure S1D; Pearson's rho $=044, \mathrm{p}=0.002$ ). Taken

14 together, these data suggest that subjects experienced and were aware of

15 performance advantages for randomly spaced stimuli, but that the extent of these

16 advantages differed across individuals.

18 To better understand these performance advantages, we tested the extent to which

19 trial-to-trial accuracy and confidence scores depended on stimulus clustering within

20 the randomly spaced stimulus arrays. Specifically, we computed within-cluster

21 variance (WCV) for each color array (as for the binary model) to evaluate whether

22 this clustering statistic could be used to predict subjects' accuracy of, and confidence

23 in, color reports. As predicted by chunking models (figure 3I), subjects were more 
1 accurate for low WCV trials; performance on high WCV trials was similar to that in

2 the fixed spacing configuration (figure 4A). Furthermore, subject wagering also

3 decreased monotonically with WCV, such that betting behavior on the highest WCV

4 (least clustered) color arrays was similar to that on fixed spacing trials (figure 4B).

Performance advantages are not due to binding errors.

8 An alternative explanation of these effects is that subjects exhibited higher

9 performance in low WCV trials simply because they committed binding errors (Bays

10 et al., 2009), mistaking one color for a nearby related color. To address this issue,

11 we applied a generalized linear model (GLM) to the binary accuracy and confidence

12 data. In particular, we included the distances between the target color and each non-

13 probed color as nuisance variables to determine whether the apparent WCV effects

14 could be better explained by a tendency to report non-probed colors, which are

15 often closer to the target color for more clustered stimulus arrays. When this model

16 was applied separately to subject accuracy and accuracy of reports simulated from a

17 mixture model that included binding errors, coefficients for WCV were negative in

18 fits to subject data but negligible when fit to simulated data, suggesting that

19 performance improvements mediated by WCV were not simply a reflection of

20 binding errors (figure S2; subject accuracy $\beta=0.028, t=5.3, p<10 e-6$ ).

21 Furthermore, when the same model was fit to post-decision wagers, coefficients for

22 WCV took similarly negative values, suggesting that subjects were aware of the 
1 performance advantages that they gained from the clustered stimulus arrays $(\beta=-$

$2 \quad 0.049, \mathrm{t}=-5.7, \mathrm{p}<10 \mathrm{e}-7)$

3

$4 \quad$ Trial-by-trial adjustment in accordance with reinforcement learning

5 The GLM also included terms to account for other factors that could potentially

6 affect task performance, including feedback from previous trials. Positive feedback

7 on the previous trial was predictive of higher accuracy and confidence for the

8 current trial, in a manner consistent with trial-by-trial behavioral adjustment

9 (figure S2, “correct ( $t-1$ )" coefficient; accuracy $\beta=0.017, t=5.0, p<10 e-6$;

10 confidence $\beta=0.026, t=4.9, p<10 e-5)$. This predictive relationship was unlikely to

11 be driven by autocorrelation in performance, as such an explanation should also

12 predict that confidence measurements relate to accuracy on future trials, and this

13 relationship was not evident in the data (figure $S 2$, "correct $(t+1)$ " coefficient;

14 confidence $\beta=0.0017, t=0.3, p=0.75$ ). Despite seemingly robust feedback-driven

15 effects, overall performance improvements across the session were somewhat

16 modest, as evidenced by a relatively small positive coefficient for a term in the GLM

17 relating block number to accuracy (figure S2, "block" coefficient; accuracy $\beta=0.007$,

$18 \mathrm{t}=2.3, \mathrm{p}=0.02$, Bayes Factor $=2.3$ ). Thus, the GLM results suggest that subjects

19 gained a performance advantage for clustered target arrays, modulated behavior in

20 response to previous feedback, and improved slightly over the course of task

21 sessions. Below we provide a more specific test of whether subjects were adjusting

22 chunking strategies in accordance with the reinforcement learning strategy

23 employed in the binary encoding model. 
bioRxiv preprint doi: https://doi.org/10.1101/098939. this version posted November 2, 2017. The copyright holder for this preprint (which was not certified by peer review) is the author/funder, who has granted bioRxiv a license to display the preprint in perpetuity. It is made available under aCC-BY-NC-ND 4.0 International license.

Nassar et al

A

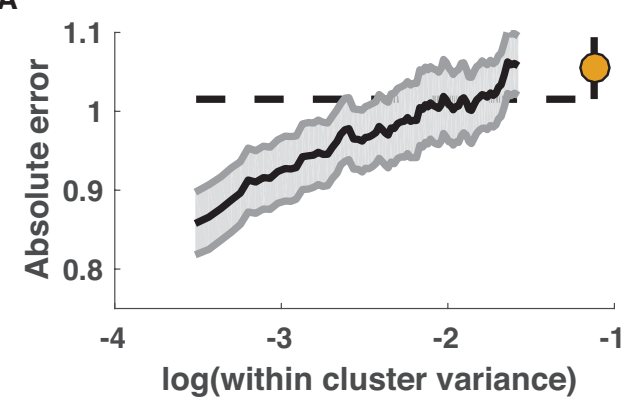

B

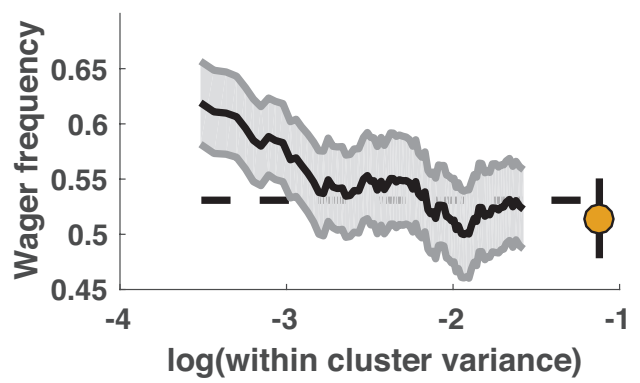

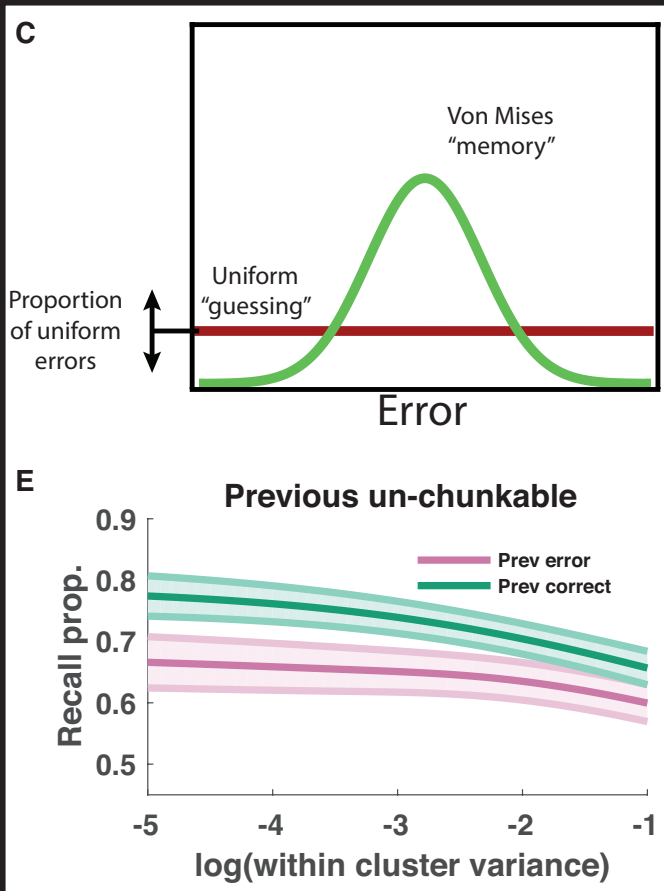

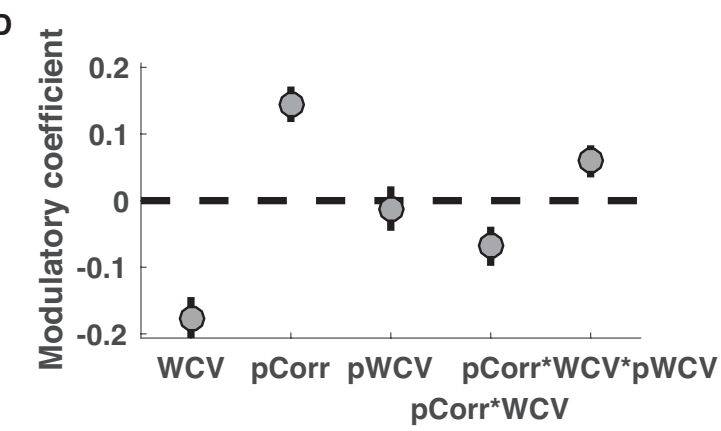

$\mathbf{F}$

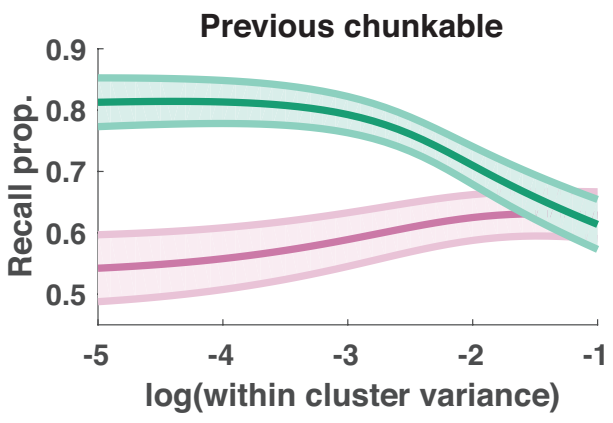




\section{$1 \quad$ Figure 4: Memory recall and confidence are enhanced for clustered stimulus arrays and}

2 adjusted according to trial feedback in accordance with model predictions. A\&B: Memory

3 performance and confidence increase with stimulus clustering. Mean absolute error magnitude (A)

4 and high wager frequency (B) were computed per subject in sliding bins of within-cluster variance

5 (larger values = decreased stimulus clustering) for random (lines) and fixed spacing conditions

6 (points). Lines and shading reflect mean and SEM across subjects. C-F: Mixture model fits reveal

7 recall benefit of stimulus clustering and hallmark of feedback-driven criterion adjustments. C:

8 Subject data were fit with a mixture model that considered reports to come from a mixture of

9 processes including 1) a uniform "guess" distribution, 2) a "memory+binding" distribution centered

10 on the color of the probed target, and 3) a "binding error" distribution including peaks at each non-

11 probed target [not shown]. Additional terms were included in the model to allow the recall

12 probability to vary as a logistic function of stimulus clustering, recent feedback, and their interaction.

13 D-F: Recall probability was modulated by feedback and stimulus clustering in a manner suggestive of

14 trial-to-trial adjustments of chunking. Mean/SEM coefficients across subjects for each modulator of

15 recall (log within-cluster variance (WCV), previous trial feedback (pCorr), previous trial log within-

16 cluster variance (pWCV), pCorr*WCV and pCorr*WCV*pWCV) are represented from left to right as

17 points/lines. Multiplicative interaction terms were included to capture the form of criterion

18 adjustments that were used to facilitate criterion learning in the binary encoding model (Fig 3G-I).

19 E\&F: Recall probability of best-fitting descriptive models plotted as a function of the log within-

20 cluster variance for the current trial and divided according to previous feedback (color) and the log

21 within-cluster variance from the previous trial [E: pWCV =-1, F: pWCV=-5]. Lines/shading reflect

22 mean/SEM across subjects. Feedback effects are consistent with reinforcement-learning as

23 implemented in the binary encoding model: when chunking clustered stimulus arrays is rewarded

24 with positive feedback, it is reinforced, leading to selective performance improvements for clustered

25 stimulus arrays on the subsequent trial. 
1 To better understand how working memory fidelity depends on stimulus clustering

2 and feedback history, we extended a basic mixture model of subject memory reports

3 (figure 4C; (Bays et al., 2009; Zhang \& Luck, 2008)). The model considers memory

4 reports to come from a mixture distribution that includes a "memory" component

5 centered on the probed color, a "guessing" component uniformly distributed across

6 color space and a "binding error" component that assumes reports are centered on

7 non-probed target colors (not shown in figure; (Bays et al., 2009; Zhang \& Luck,

8 2008)). We allowed the proportion of recall (1-guessing) to vary as a function of the

9 key factors that should affect performance if subjects are optimizing chunking.

10 Across subjects, the probability of recall increased with stimulus clustering, as

11 assessed by within-cluster variance (figure 4D; $t=-5.7, p<10 e-6$ ). Notably,

12 performance improvements due to stimulus clustering were observed even for

13 stimulus arrays in which the probed target color was dissimilar to the cluster of

14 non-probed target colors (figure S3), as predicted by the binary encoding model

15 (i.e., where chunking increases resources and probability of encoding of other

16 items). Together with the GLM results above, these findings rule out alternative

17 explanations in which WCV effects would arise simply by mistaking one color for

18 another one nearby, and instead support our thesis that clusters of stimuli can be

19 stored jointly to conserve representational space.

21 Furthermore, additional coefficients provided evidence that people adjusted

22 chunking strategies online as a function of reward feedback in a manner similar to

23 that used to optimize performance in the binary encoding model (figure 3G). In 
1 particular, in our reinforcement learning implementation, the partitioning criterion

2 was adapted based on the amount of chunking in the previous trial and the

3 concomitant reward feedback, and it selectively contributed to performance

4 improvements for the most clustered stimulus arrays (figure $3 \mathrm{H}-\mathrm{F}$ ). To explore the

5 possibility that people adjust chunking in a similar way, we included additional

6 terms in the mixture model to allow recall probability to vary as a function of

7 previous trial feedback (pCorr), proxies for previous and current trial clustering

8 (pWCV, WCV), and their predicted interactions (see Methods). The best-fitting

9 coefficients revealed an overall recall bonus on trials following correct feedback

10 (pCorrect: $\mathrm{t}=5.4, \mathrm{p}<10 \mathrm{e}-5$ ), but also that the magnitude of this bonus was greater

11 for trials in which stimuli were more clustered (pCorrect * WCV: $t=-2.1, p=0.04$,

12 Bayes Factor $=1.6$ ) and for trials in which the level of stimulus clustering matched

13 that of the previous trial (pCorrect $*$ WCV $*$ pWCV: $t=2.1, p=0.04$, Bayes Factor $=$

14 1.6; figure 4D). Consistent with optimization of chunking via reinforcement learning,

15 these interactions capture a tendency toward larger feedback-driven changes in

16 task performance when both the current and previous trial color arrays were highly

17 clustered (figure 4E\&F). Taken in the context of our model, this suggests that when

18 subjects are rewarded for successfully chunking a stimulus array, they are more apt

19 to repeat this chunking on the subsequent trial. Moreover, these strategic

20 adjustments rule out an obligatory alternative mechanism in which chunking occurs

21 regardless of task demands. 
1 In sum, our abstract model of a memory-limited system predicted performance

2 advantages and trial-to-trial adjustments associated with selective chunking that

3 were subsequently validated in empirical data from human subjects performing a

4 working memory task. Nonetheless, the abstract system that we explored does not

5 mimic the processing architecture that is used by the brain, leaving open the

6 question of how selective chunking might be achieved using biological hardware. In

7 the next section we consider this question in depth, by attempting to endow a

8 biologically inspired active maintenance model of working memory with the

9 computational elements necessary to achieve the chunking-based performance

10 bonus predicted by the abstract model and observed in data from human subjects.

11

A

Local recurrent excitation

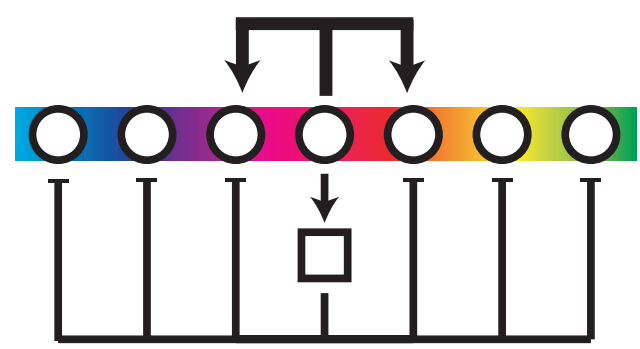

Uniform lateral inhibition

C

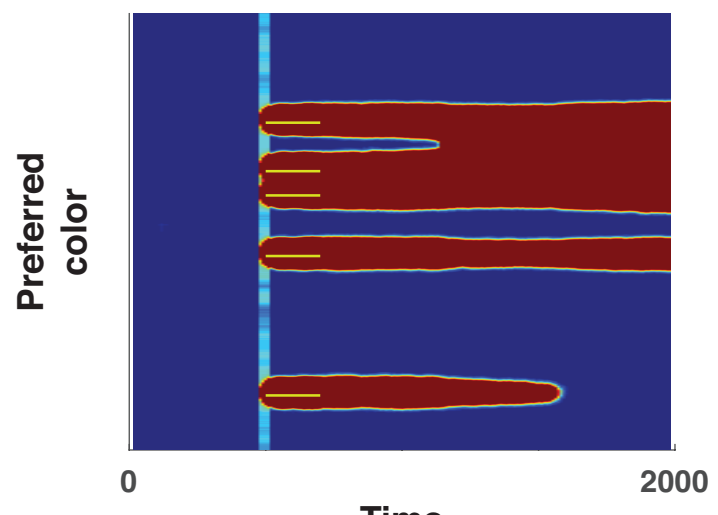

12

Time
B

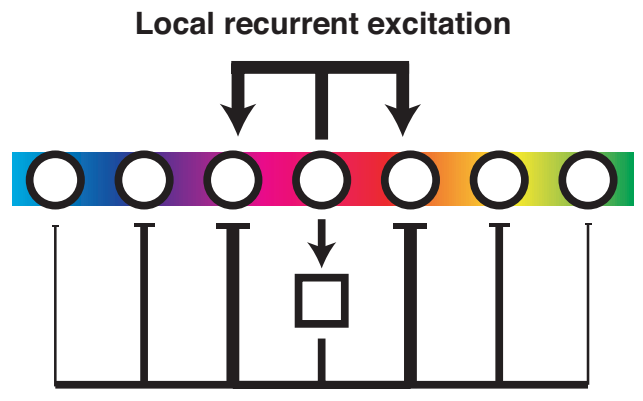

Broadly tuned lateral inhibition

D

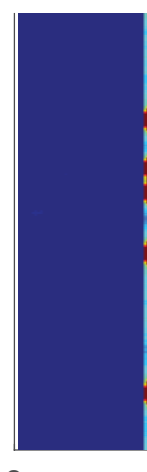

0

Time
Firing rate $(\mathrm{Hz})$

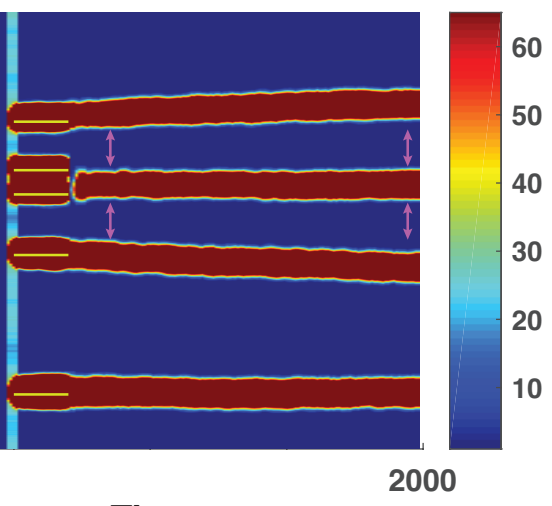

50

40

30

20

10 
3 Figure 5: Center-surround connectivity as a mechanism to support chunking and partitioning

4 operations needed to optimize working memory storage. A\&B) Local recurrent excitation and

5 lateral inhibition are critical for active working memory maintenance in biologically plausible neural

6 networks (Almeida et al., 2015; Wei et al., 2012). However, the exact form of lateral inhibition has

7 been varied across studies, with the most common version employing uniform inhibition across the

8 entire population of tuned excitatory neurons (A, (Wei et al., 2012)) whereas others employ broadly

9 tuned inhibition such that similarly tuned excitatory neurons indirectly exert stronger inhibitory

10 forces on one another (B, (Almeida et al., 2015)). C) Simulated firing rates (redder colors indicate

11 greater firing) of a population of color tuned neurons using the connectivity architecture described in

12 panel A performing a working memory task (ordinate reflects neural tuning preference; abscissa

13 reflects time in milliseconds; yellow bars indicate $200 \mathrm{~ms}$ color inputs delivered in a fixed pattern

14 across network architectures). As described by Wei and colleagues, bumps of neural activity

15 sometimes collide, producing "merged" representations (e.g., top activity bump in panel C), a possible

16 mechanism for chunking. However, also as described by Wei and colleagues, collisions are somewhat

17 indiscriminate and can increase overall population firing, which in turn can lead to collapse of other

18 activity bumps (e.g., bottom activity bump) and hence forgetting. D) Simulated firing rates from the

19 same population of neurons for the same task, but using center-surround connectivity (i.e., broadly

20 tuned inhibition). Note that the closest bumps of activity are selectively chunked (e.g., second and

21 third bump from top), but the tuned inhibition effectively partitions more distantly separated

22 representations (e.g., the top from the second and third) and prevents forgetting of unrelated items.

23 A related consequence of the tuned inhibition is that partitioned representations exert repulsive

24 forces on one another during the delay period (see differences in separation of activity bumps at pink

25 arrows). Thus, tuned inhibition affords selective partitioning of representations, but changes

26 representations through inter-item repulsion. 
Center-surround dynamics as a mechanism for chunking and partitioning

4 The brain is thought to implement visual working memory in neural networks that

5 include individual neurons tuned to specific visual features and capable of

6 maintaining representations in the absence of input (Curtis \& D'Esposito, 2003;

$7 \quad$ Fuster \& Jervey, 1981; Goldman-Rakic, 1995; E. K. Miller \& Cohen, 2001; Warden \&

8 Miller, 2007). In computational models, the ability of a neural network to maintain

9 feature representations in the absence of inputs depends critically on the recurrent

10 connections between neurons (Barak, Sussillo, Romo, Tsodyks, \& Abbott, 2013;

11 Durstewitz \& Seamans, 2002; Kilpatrick, Ermentrout, \& Doiron, 2013; Murray et al.,

12 2014; X. J. Wang, 1999). In particular, persisting feature representations, like those

13 of the colors in our task, are facilitated by local excitatory connections between

14 similarly tuned neurons and by broad inhibition between neurons (X. J. Wang,

15 1999). Importantly, for simplicity, the most common variant of such models

16 includes uniformly weighted connections for inhibitory neurons (figure 5A). While

17 this model exhibits merging of color representations and hence provides a

18 promising mechanism for chunking, it produces promiscuous merging of individual

19 color representations (bumps), large and unwieldy bumps of population activity,

20 and due to uniform lateral inhibition, forgetting of further colors (figure 5C; (Wei et

21 al., 2012)). Thus in a sense, such bump collisions are analogous to the chunking

22 implemented in our more abstract binary encoding model, yet they lack the

23 selectivity necessary to mediate performance optimization, and indeed, predict the 
1 opposite pattern of performance than seen empirically, with worse performance for

2 randomly spaced arrays and improved performance for fixed arrays (Wei et al.,

3 2012)

4

5 We considered whether other patterns of connectivity would remedy this issue.

6 Notably, physiological data suggest that neural responses within such networks

7 obey center-surround receptive field architectures that are present throughout the

8 visual system (Hubel \& Wiesel, 1959; 1965), are supported by lateral connectivity

9 (Ben-Yishai, Bar-Or, \& Sompolinsky, 1995; Kohonen, 1982; Somers, Nelson, \& Sur,

10 1995), and predict biases previously observed in visual working memory reports

11 (Almeida et al., 2015; Kiyonaga \& Egner, 2016). We thus altered the Wei et al. model

12 to include broadly tuned inhibition in accordance with center-surround recurrence,

13 whereby Feedback inhibition is stronger for neurons with similar color tuning

14 (figure 5B). In this case, recurrent excitation promotes merging of nearby color

15 representations, but tuned inhibition prevents the merged representation from

16 expanding in width, thereby preventing it from 1) suppressing other activity bumps

17 through an overpowering lateral inhibition leading to forgetting, and 2)

18 indiscriminately merging with other activity bumps (figure 5D). Indiscriminate

19 merging is prevented through repulsive forces exerted by each activity bump on

20 neighboring representations, which enable separation of dissimilar representations

21 (see widening gap noted by pink arrows). Thus, center-surround recurrence

22 provides a dynamic analog to the selective chunking necessary for optimization in

23 the binary encoding model; nearby representations that share excitatory recurrent 
1 connections are merged into a single representation (chunking) whereas more

2 distantly separated representations are repulsed by tuned inhibition to effectively

3 partition moderately dissimilar color representations (partitioning).

5 This center-surround connectivity profile promotes complex item interactions that

6 can be summarized by a "difference of Gaussians" function that mediates the

7 attraction and joint representation of similar colors and the repulsion of dissimilar

8 ones (figure 6A; yellow shading). If the two stored colors are similar enough to

9 promote mutual recurrent excitation, each represented color will experience biased

10 excitation in the direction of its neighboring color, and eventually the two color

11 "bumps" will merge to form a single representation (figure 6A) (Wei et al., 2012). In

12 contrast, if the stored colors are separated beyond the narrowly tuned recurrent

13 excitation function, mutual recurrent inhibition will dominate, leading to a net

14 repulsion of color representations from one another (Felsen, Touryan, \& Dan, 2005;

15 Kiyonaga \& Egner, 2016), which can serve as a selective partition (figure 6A). 


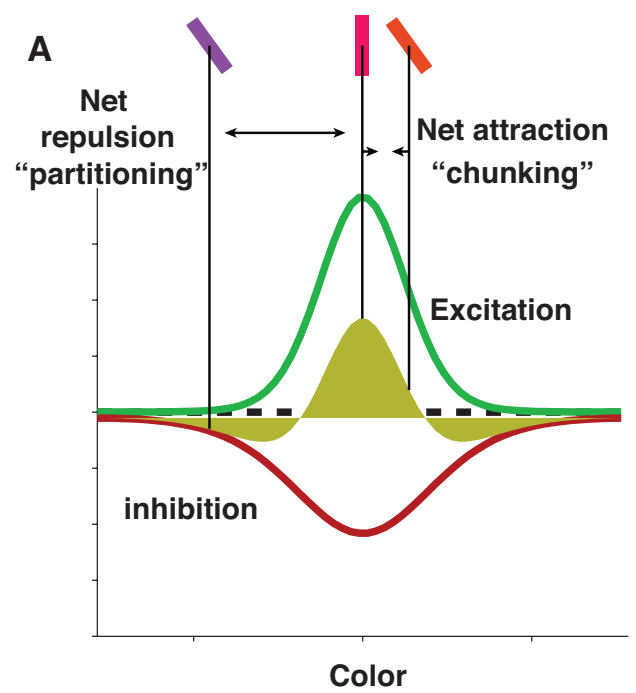

C

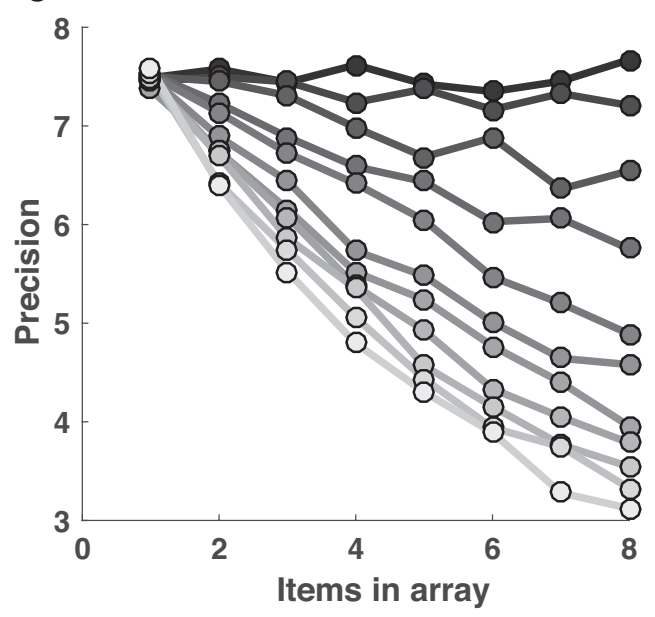

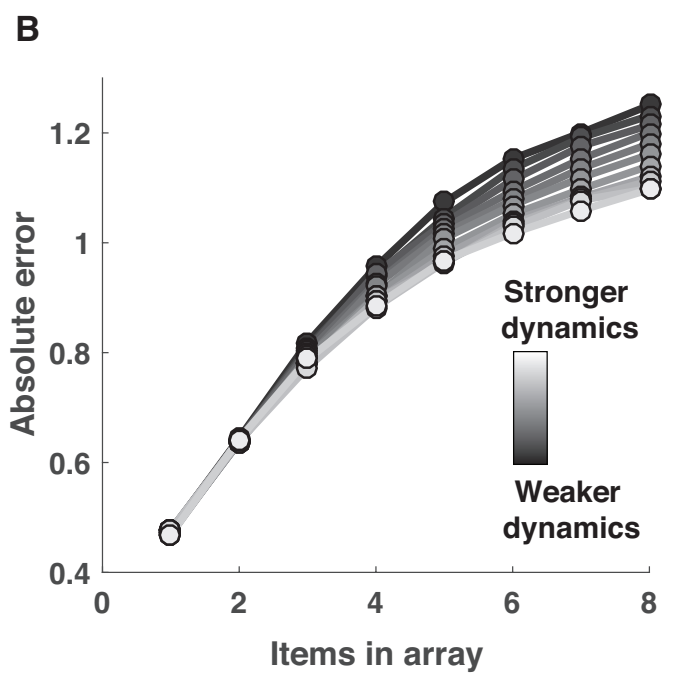

D

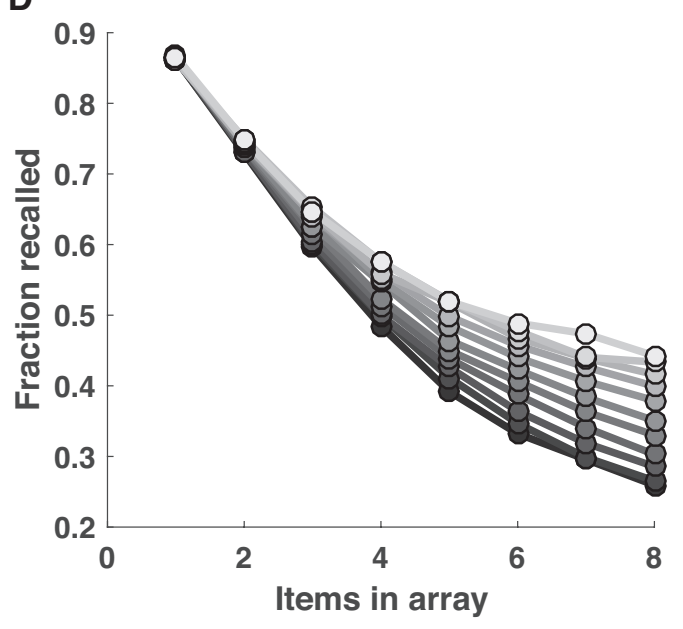

2 Figure 6: Center-surround dynamics facilitate attractive and repulsive inter-item forces that

3 can improve recall at the cost of precision. A) Local recurrent excitation and broadly tuned lateral

4 inhibition give rise to two counteracting forces: recurrent excitation facilitates attraction of

5 neighboring representations through "bump collisions" (Wei et al., 2012), whereas broadly tuned

6 lateral inhibition facilitates repulsion of distinct bumps of neural activity (Felsen et al., 2005;

7 Kiyonaga \& Egner, 2016). Together, these forces produce a difference of Gaussians tuning function

8 (yellow shading) that facilitates attraction of closely neighboring representations but repulsion of

9 more distant ones. Here we model these effects at the cognitive level by assuming that two imprecise

10 internal representations of color are chunked, and jointly represented by their mean value, with a

11 fixed probability defined by a narrowly tuned von Mises distribution (green curve; B\&C) in order to

12 mimic the effects of narrowly tuned excitation. After probabilistic chunking, each color 
1 representation exerts a repulsive influence over all other representations with a magnitude defined

2 by a broadly tuned von Mises distribution (red curve) in order to mimic the effects of broadly tuned

3 inhibition. The model stores a Poisson number of the representations, chunked or otherwise, for

4 subsequent recall. B) The influence of center-surround dynamics over model performance can be

5 manipulated by applying a gain to the amplitude of the excitation and inhibition functions such that

6 larger values correspond to greater item interdependencies and lead to smaller errors on average

7 (lighter colors correspond to higher gain). C\&D) The performance improvement mediated by

8 increasing center-surround dynamics relies on a tradeoff between recall probability and precision,

9 through which increased attractive and repulsive forces reduce precision (lighter bars; C), but

10 enhance recall probability (lighter bars; D).

13 We next examined how chunking, as implemented through this framework, would

14 impact performance as a function of stimulus array size. To do so, we summarized

15 the impact of center-surround connectivity in an algorithmic model of working

16 memory performance that contained a small number of intuitive parameters. We

17 implemented attractive and repulsive forces among stored memories in accordance

18 with narrowly tuned excitation and broadly tuned inhibition functions (see Methods

19 for details). On each trial, each color from the target array was: 1) perturbed by a

20 mean zero random variable to simulate neural noise, 2) chunked with each other

21 color in the array with probability proportional to the excitation tuning function, 3 )

22 repulsed by each other color in the array with magnitude proportional to the

23 inhibition tuning function, and 4) probabilistically stored across the delay period

24 according to a Poisson process. The proportionality constants allowed us to examine

25 the performance of models ranging from those that were not affected by recurrent 
1 dynamics (zero proportionality constant) to those that were highly affected (large

2 proportionality constant).

3

4 Models implementing greater recurrent dynamics achieved better performance

5 through a recall/precision tradeoff. Performance was simulated on delayed report

6 tasks in which target number (array size) ranged from one to eight. Performance of

7 models employing recurrent dynamics was slightly worse for easier tasks but

8 dramatically improved for more difficult ones, similar to the effects observed in the

9 binary model above (figure 6B; lighter lines represent stronger recurrent

10 dynamics). Here, though, performance differences were characterized by opposing

11 effects of recurrent dynamics on precision and recall. Models employing recurrent

12 dynamics showed improved recall, particularly in the hardest tasks, as attractive

13 forces allowed for the storage of multiple target features in a single representation

14 (figure 6D). However, these same recurrent dynamics came at the cost of reduced

15 precision, as both attractive and repulsive forces reduced the fidelity of stored color

16 representations (figure 6C). In standard models of resource limitations, precision

17 decrements with increased array sizes have been attributed to the division of a

18 limited resource. However, in the recurrent dynamics models, the decrement in

19 precision is caused by the increase in inter-item interactions that occurs when

20 additional items are added to the memory array. Thus, the inclusion of recurrent

21 dynamics affects the nature of capacity limitations: minimizing the impact of center-

22 surround forces leads to a specific decay in recall as a function of array size, as

23 predicted by "slots" models, whereas maximizing the impact of center-surround 
1 forces leads to decays in precision across set size, which is a key feature of resource

2 depletion accounts (Bays \& Husain, 2008; Luck \& Vogel, 2013; Ma et al., 2014; Zhang

3 \& Luck, 2008).

5 In summary, inter-item dependencies that emerge from center-surround dynamics

6 are sufficient to mediate the performance bonuses of chunking, but do so at the cost

7 of precision. Thus, if working memory is optimized through chunking in this way, it

8 should lead to a higher probability of recall for colors of clustered stimulus arrays

9 but more precise recall of colors for less clustered ones. In principle, such

10 optimization could be guided in cognitive or real-world tasks by implicit or explicit

11 feedback to favor successful chunking strategies and avoid unsuccessful ones.

13 People are less precise when remembering clustered target arrays.

15 Our center-surround implementation of chunking and partitioning predicts that

16 chunking advantages should come at the cost of precision (figure 6B\&C). To test this

17 prediction, we examined the difference in error distributions for random vs. fixed

18 spacing, pooled across all subjects (figure 7, left column). The error distributions

19 from both conditions were consistent in shape with those previously reported in

20 similar tasks (figure 7A\&D) (van den Berg et al., 2014). However, the error

21 distributions differed subtly between the two conditions: in the random-spacing

22 condition, subjects made more moderately small errors, but did not have more

23 perfect recalls (figure 7G). This pattern of difference was also evident in data 
1 simulated from the center-surround chunking model (figure 7, middle column) but

2 not in data simulated from an independent encoding model fit to subject behavior

3 (figure 7, right column). Thus, both the subjects and the center-surround chunking

4 model reported more colors that were slightly offset from the target color in the

5 random-spacing condition than in the fixed-spacing condition, consistent with a

6 reduction in precision resulting from chunking.
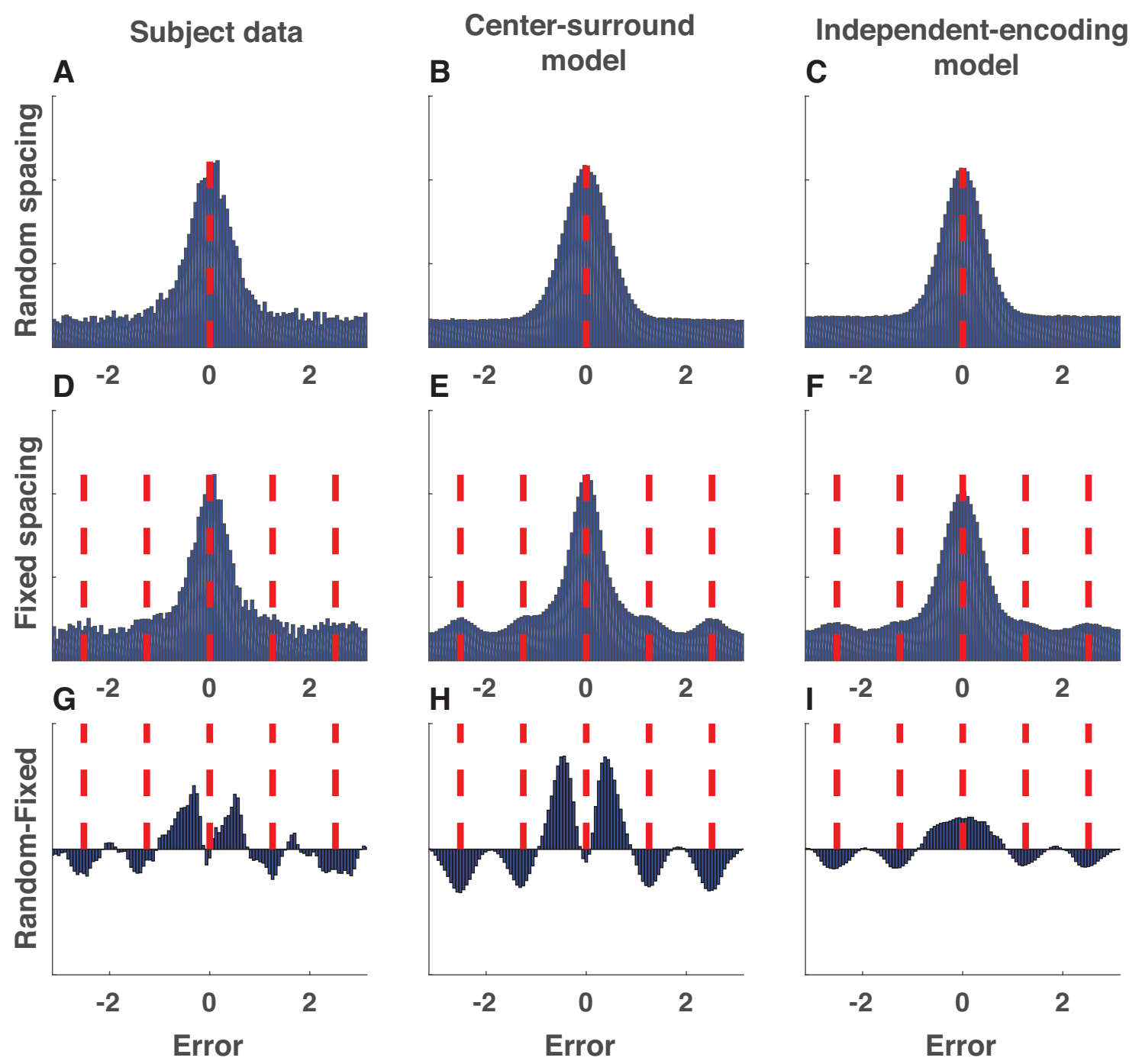

8 Figure 7: Error distributions reveal evidence for center-surround chunking. A-C) Signed color

9 reproduction errors made in the random spacing condition by (A) subjects, (B) center-surround

10 chunking models, and (C) independent encoding models. Data is collapsed across all simulated or 
1 actual sessions. D-F) Same as A-C but for the fixed spacing condition. Red dashed lines indicate

2 probed and non-probed target locations. Note that the alignment of non-probed target locations

3 emphasizes the prominence of non-probed target reports (binding errors), which would appear

4 uniformly distributed in the random spacing condition. G-I) Difference in above error distributions

5 for random minus fixed. To aid in visualization, bin count differences were smoothed with a Gaussian

6 kernel (standard deviation $=1$ bin). Subjects and the center-surround chunking model show

7 increased moderately small, but non-zero, errors in the random spacing condition. Note that

8 differences of reports between the random and fixed conditions near the non-probed targets are

9 present in both models, as they simply reflect an artifact of the alignment of binding errors in the

10 fixed spacing condition.

11

12 Errors are modulated by nearest neighbors consistent with chunking via recurrence

14 To better understand the nature of these error distributions, and to what extent

15 they are predicted by attraction and repulsion forces in center-surround dynamics,

16 we sorted trials according to the non-probed target color that was most similar to

17 the probed target color (nearest neighbor color; see Methods for details). This

18 procedure revealed structure in individual subject color reports related to the

19 nearest neighbor non-probed color (see figure S4). To determine whether such

20 structure persisted systematically across subjects, we fit a descriptive mixture

21 model to error distributions pooled across subjects in sliding windows of nearest

22 neighbor distance. The model contained free parameters to examine 1) the

23 precision of error distributions, 2) the bias of error distributions toward (or away

24 from) the nearest neighbor non-probed target color, and 3) the relative proportion 
1 of trials that were recalled, forgotten, or mis-bound (in keeping with nomenclature

2 from previous literature (Bays et al., 2009; Fallon, Zokaei, \& Husain, 2016)).

5 The model fits revealed that subject precision and bias depended on neighboring

6 colors in a manner consistent with chunking through recurrent dynamics. In

7 particular, subject memory reports were biased towards the nearest neighbor color

8 if it was sufficiently similar to the probed target color, but biased away from it if it

9 was sufficiently dissimilar (figure 8A). This pattern of bias maps onto the idea of a

10 narrowly tuned excitation function promoting attraction of nearby targets and a

11 broadly tuned inhibition function promoting repulsion of more distant ones (see

12 figure 8A). Precision also depended on nearest neighbor color distance: subject

13 precision was maximal when the nearest neighbor color was most dissimilar to the

14 probe color and minimal when it was moderately similar (figure 8D). In addition,

15 fits revealed an increase in the proportion of correct recalls, and a corresponding

16 decrease in the number of uniform guesses, when a nearby neighbor color existed in

17 the stimulus array (figure S5). This pattern of results was strikingly consistent with

18 those produced by a chunking model based on recurrent dynamics (figure 8 , middle

19 column) but not with those produced by the best-fitting mixture model (figure 8,

20 right column). 
Subjects

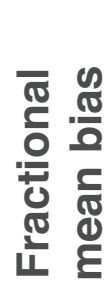

$-0.2$

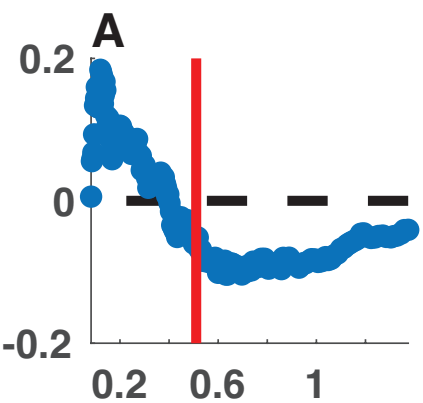

$\frac{\text { 듬 }}{\frac{0}{0}}$

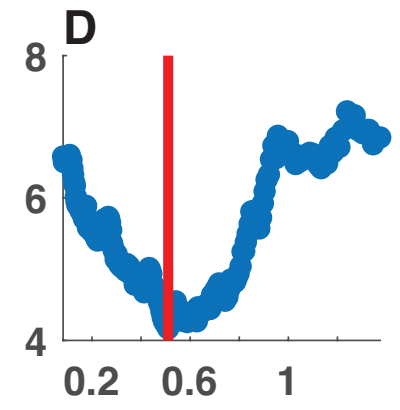

Nearest neighbor distance
Center-surround
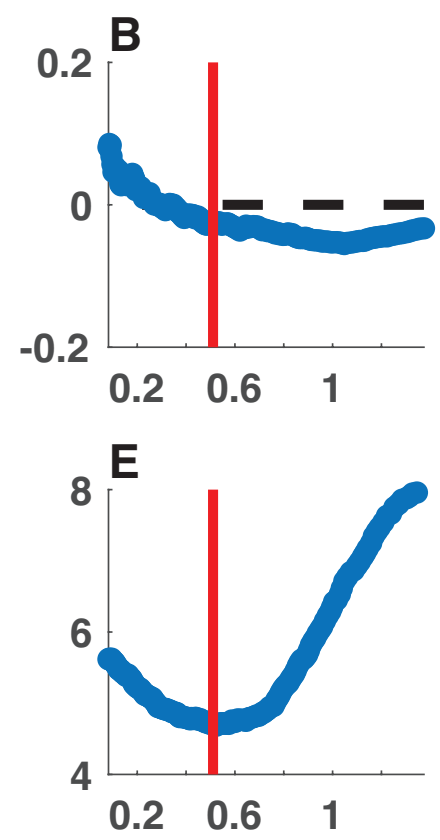

Nearest neighbor distance
$-0.2$

Independent
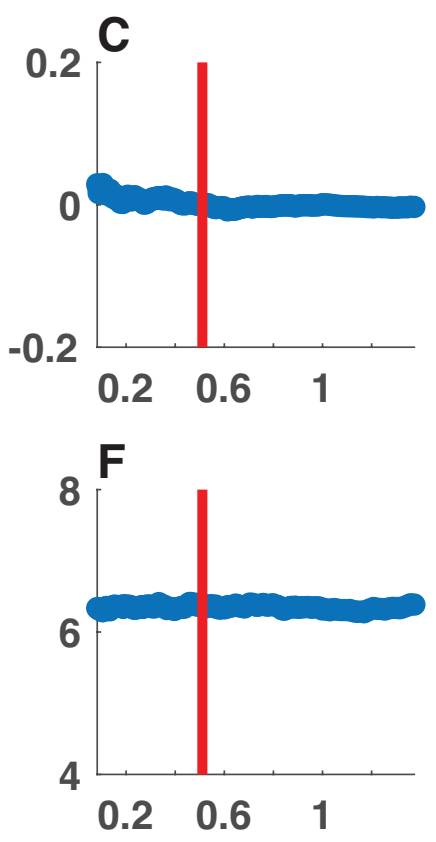

Nearest neighbor distance

Figure 8: Neighboring stimulus features affect bias, precision, and recall probability as

predicted by the center-surround chunking model. Subject (left) and simulated (center = center-

surround, right $=$ independent encoding) data were collapsed across all sessions and binned in

5 sliding windows according to the absolute distance between the probed target color and the most

6 similar non-probed target color (nearest neighbor distance; abscissa). Data in each bin were fit with a

mixture model that included free parameters to estimate 1) the bias of memory reports towards the

closest color in the target array expressed as a fraction of distance to that target (A-C), and 2) the

precision of memory reports (D-F). The qualitative trends present in subject data are also present in

10 data simulated from the center-surround chunking model but not in those simulated from the

11 independent encoding model. Red bars reflect the nearest neighbor distance at which precision fits

12 to subject data were minimal and also corresponds well with the crossover point of the bias fits. 
1 Quantitative model-fitting of center-surround model reveals empirical evidence of

2 performance advantage for chunking.

3

4 While the above mixture model fits revealed structure across subjects on average,

5 here we provide a quantitative fit of the center-surround model directly to the trial-

6 by-trial memory report data for each individual subject, allowing us to quantify

7 chunking effects and examine the range of behavioral chunking strategies across

8 individual participants. To make this fitting more tractable (i.e., to facilitate a closed

9 form likelihood function), we simplified the center-surround model while retaining

10 its core elements. These simplifications included removal of sensory noise and the

11 simplification of the center (chunking) and surround (repulsion) forces (see

12 Methods). We fit three different models to estimate the potentially separable

13 contributions of center and surround functions using maximum likelihood. The

14 "center" model estimated the partitioning criterion, which summarized the center

15 function, as a free parameter, whereas the "surround" model fit the repulsion

16 coefficient as a free parameter. The "center-surround" model fit both center and

17 surround with free parameters. All models were also compared to a basic mixture

18 model. Goodness of fit was evaluated for each model using AIC, which applies a fixed

19 complexity penalty for each parameter and provided better model recovery for

20 simulated data than BIC.

22 Comparison of the center-surround model to a basic mixture model revealed an

23 explanatory advantage of the former, albeit with considerable heterogeneity across 
1 individual subjects. The center-surround model achieved the lowest mean relative

2 AIC values of all models (mean[SEM] relative AIC: basic mixture $=4.9[0.9]$, center $=$

$35.5[0.8]$, surround $=4.1[0.7]$, center-surround $=2.9[0.6])$. Inclusion of both center

4 and surround terms was favored by a likelihood ratio test $\left(\chi^{2}(94)=281, p<10 e-5\right)$

5 and Bayesian model selection favored the center-surround model in terms of

6 explaining the largest proportion of subject behavior (exceedance probability =

7 0.85). However, the best-fitting model was not consistent across subjects, with some

8 subjects best fit by the simple mixture model and others best fit by the center-

9 surround model (figure 9A). Parameter estimates from the best-fitting center-

10 surround model were also indicative of heterogeneity. For a large number of

11 subjects, the best-fitting partitioning criterions were near zero (indicating no

12 chunking), but partitioning criterions fit to the remainder of subjects were broadly

13 distributed (figure 9B). Best-fitting repulsion coefficients were more normally

14 distributed across subjects, tending to take small positive values, indicating a

15 tendency toward repulsion of partitioned representations (figure 9C).

17 Heterogeneity in model fits also related to overall task performance in a manner

18 suggestive of a performance advantage for chunking. Our modeling suggested that

19 criterion-based chunking could be used to reduce overall errors in a visual working

20 memory task, and the differences in model fits across our subjects offered us an

21 opportunity to test this idea. Consistent with chunking facilitating in-task

22 performance advantages, subjects fit with larger partitioning criterions and

23 repulsion coefficients achieved better performance on the task (figure 9E\&F; 
1 partitioning criterion: Spearman's $\rho=-0.54, \mathrm{p}=8.5 \mathrm{e}-5$; repulsion coefficient:

2 Spearman's $\rho=-0.39, \mathrm{p}=7.4 \mathrm{e}-3)$. Similar relationships were seen between model

3 preference and overall performance, with the subjects that were best fit by the

4 center-surround model also tending to have the lower absolute errors in the task

5 (figure 9D; Spearman's $\rho=-0.59, \mathrm{p}=1.6 \mathrm{e}-5$ ). In order to examine which parameter

6 of our model best predicts subject performance, we constructed a GLM to examine

7 the independent contributions of partitioning criterion and repulsion parameter

8 estimates on subject performance (as measured by mean absolute error) and found

9 that when accounting for both variables, only the partitioning criterion maintained

10 explanatory power, with higher partitioning criterions corresponding to lower

11 absolute error magnitudes (partitioning criterion $\beta=-0.95, \mathrm{t}=-3.2, \mathrm{p}=0.002$;

12 repulsion $\beta=0.8, \mathrm{t}=0.4, \mathrm{p}=0.7$ ). Thus, individuals who chunked the most liberally

13 also achieved the best task performance.

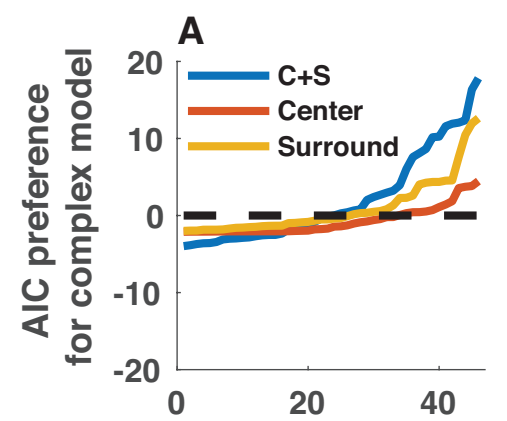

Subject - sorted by preference

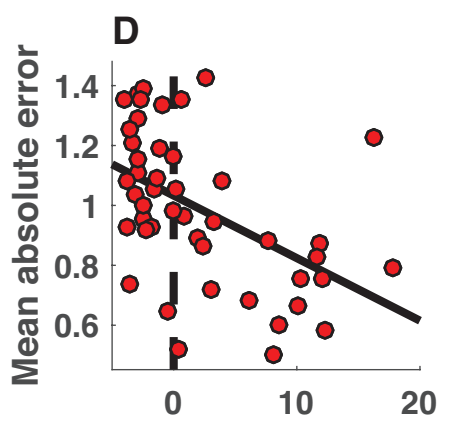

14

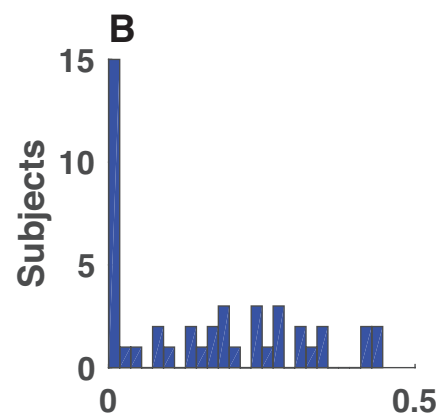

Partitioning criterion

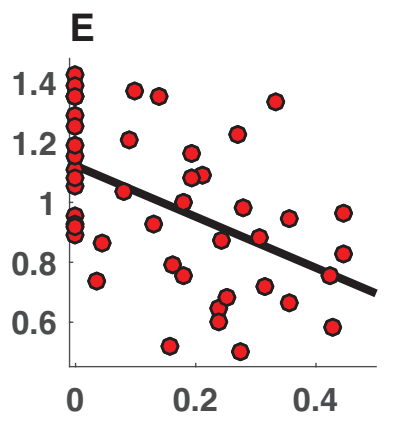

Partitioning criterion

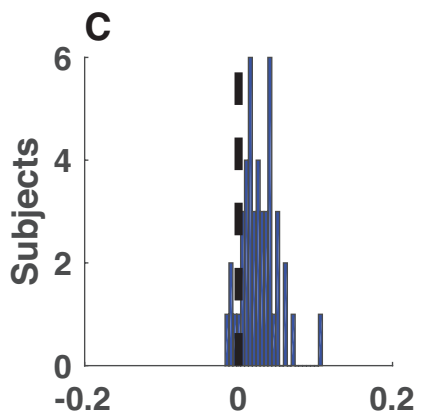

Repulsion coefficient

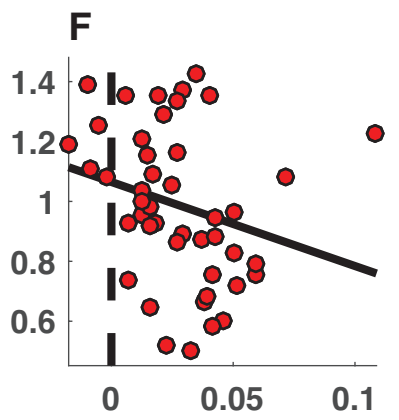

Repulsion coefficient 
2 Figure 9: Heterogeneous chunking strategies across individual subjects provide empirical

3 evidence for the performance advantages afforded by chunking. A) AIC difference between

4 simple mixture model and more complex center (orange), surround (yellow), and center + surround

5 (blue) models is plotted for each subject, sorted by model preference (positive values indicate that

6 more complex model is preferred). Aggregate AIC values favored the $\mathrm{C}+\mathrm{S}$ model, yet there was

7 substantial variability across subjects in marginal improvement afforded by the $\mathrm{C}+\mathrm{S}$ model over the

8 simpler mixture model, with AIC values providing moderate evidence for the mixture in some

9 subjects, but strong evidence for the $\mathrm{C}+\mathrm{S}$ model in other subjects. B) Partitioning criterions best fit to

10 subject data also reflected heterogeneity in strategies across subjects, with a number of subjects best

11 fit with criterion values near zero, and another subset of subjects taking values across a wider range

12 from 0.1-0.5. C) Best-fitting repulsion coefficients tended to take positive values across subjects,

13 indicating that independently represented colors tended to exert repulsive forces on one another by

14 the best-fitting model parameterization. D-F) Subjects displaying more evidence of center-surround

15 chunking performed better on the working memory task. D) Mean absolute error was greatest for

16 the subjects that displayed the least evidence of center-surround chunking, as assessed by the

17 difference in AIC between $\mathrm{C}+\mathrm{S}$ and basic mixture models $(\rho=-0.59, \mathrm{p}=1.6 \mathrm{e}-5)$. E\&F) Errors were

18 also elevated for subjects that were best fit with criterions near zero (E; $\rho=-0.54, p=8.5 e-5)$ or with

19 small or negative repulsion coefficients (F; $\rho=-0.39, p=7.4 \mathrm{e}-3$ ).

20

22 Center-surround chunking effects generalize across tasks, contribute to set-size

23 dependent changes in precision, and mediate individual differences in performance.

25 Finally, to test whether our findings were robust to changes in task conditions and

26 to examine how chunking effects vary with memory load, we fit a nested set of 
1 models to a meta-analysis dataset that included 101 subjects performing eight

2 different experiments (James M Gold et al., 2010; van den Berg et al., 2014). The

3 nested model set included models that varied in their assumptions about chunking,

4 the distributional form of error reports, and the direct effects of set size on

5 precision. The model set was built upon a base model that assumed that subjects

6 would recall a Poisson number of feature representations in the report dimension

7 and a Poisson number of features in the probe dimension on each trial, with failure

8 to recall the probe dimension resulting in a binding error and failure to recall the

9 report feature resulting in a uniform guess. Precision of memory reports was fixed

10 across trials in this base model. The nested model set included additions to the base

11 model that allowed it to account for 1) effects of center-surround chunking on the

12 represented feature value and number of stored features (C in figure 10A), 2) effects

13 of center-surround chunking on the precision of memory reports ( $\mathrm{N}$ in figure $10 \mathrm{~A}$ ),

143 ) differences in error distribution kurtosis through t-distributed memory reports

15 ( $\mathrm{T}$ in figure 10A) and 4) changes in precision as a power-function of set size (P in

16 figure 10A). Performance of the nested model set was compared to that of a variable

17 precision model with Poisson item storage and binding errors, which was the best-

18 fitting model in a recent factorial model comparison using most of the same data (VP

19 in figure 10A, (van den Berg et al., 2014)). 


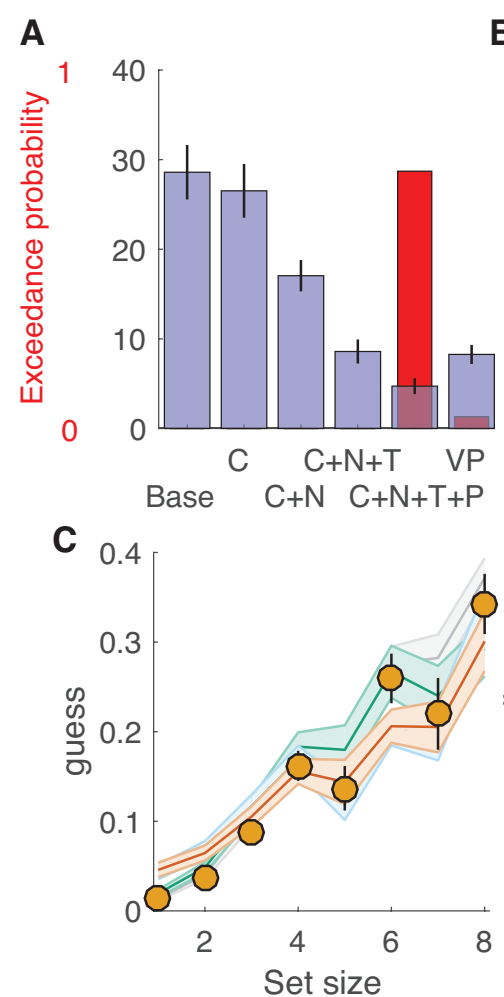

$\mathbf{F}$

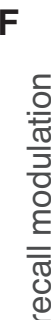

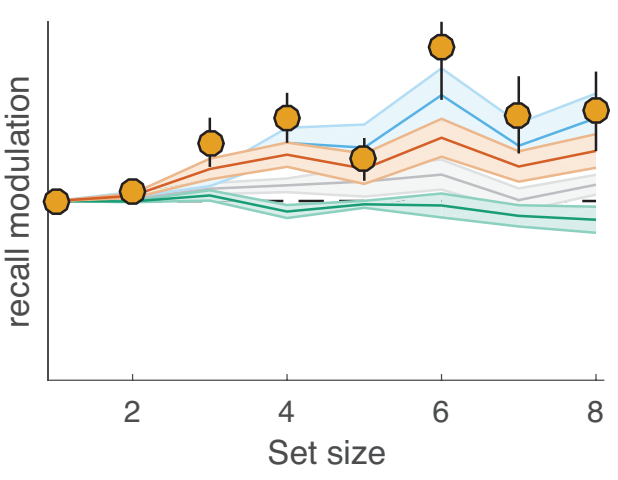

H
Van den Berg et al., 2012 (color-scroll)

Van den Berg et al., 2012(orientation)

Van den Berg et al., 2012(color-wheel)

Rademaker et al., 2012(orientation)

Wilken \& Ma, 2004

Bays et al., 2009

Zhang \& Luck, 2008

Gold et al., 2010

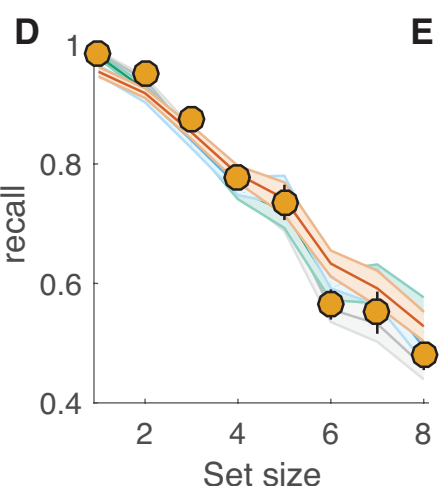

G
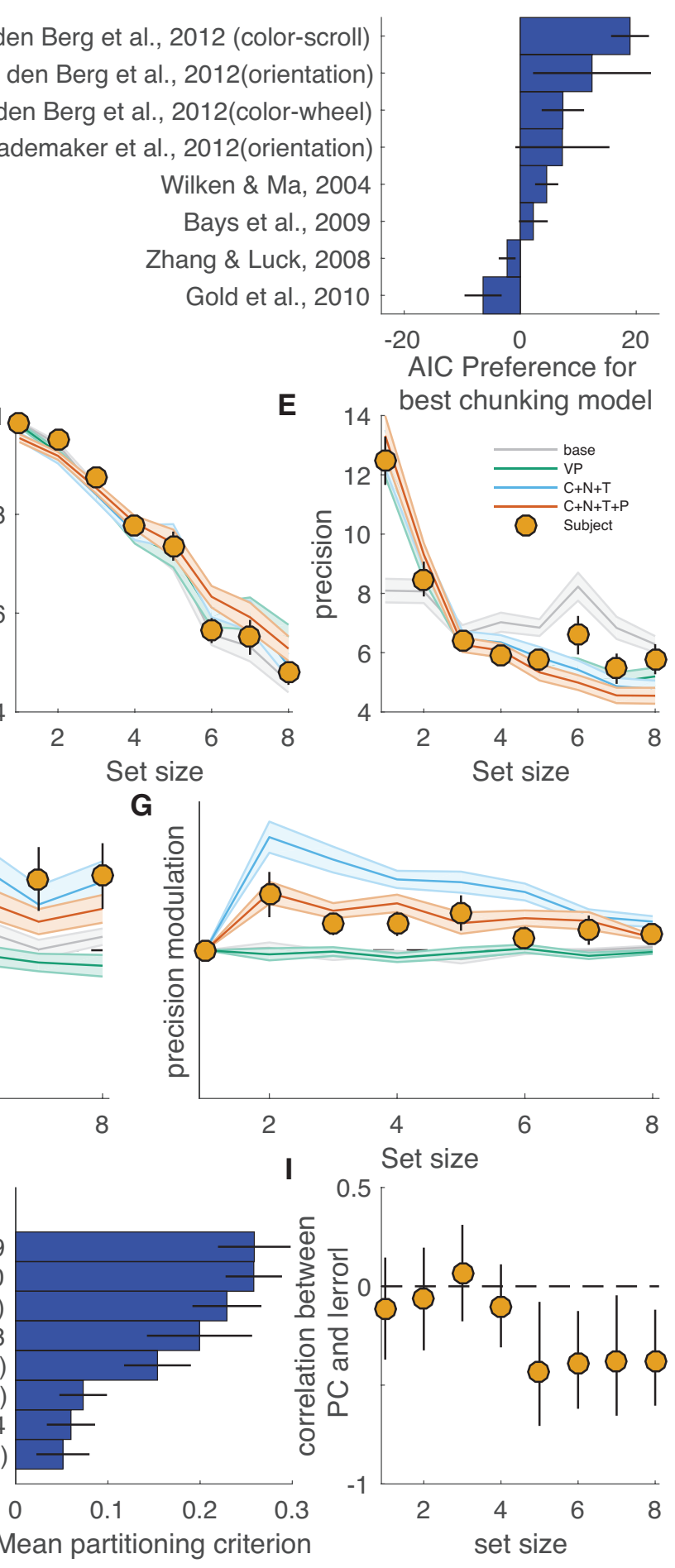

Mean partitioning criterion

Bays et al., 2009

Gold et al., 2010

Van den Berg et al., 2012 (color-scroll)

Zhang \& Luck, 2008

Van den Berg et al., 2012(color-wheel)

Rademaker et al., 2012(orientation)

Wilken \& Ma, 2004

Van den Berg et al., 2012(orientation) 
1 Figure 10: Center-surround chunking allows better fits of meta-analytic datasets and offers

2 insight into trends and individual differences in how memory degrades with set size. A) Blue

3 bars/lines represent mean/SEM relative AIC (AIC relative to that of the best model for each subject)

4 and red bars reflect exceedance probability for a nested model set. Base refers to the base model, C

5 includes center-surround chunking, $\mathrm{N}$ allows for chunking- and repulsion-induced report variability,

6 T allows for t-distributed errors, and P allows precision to vary as a power-function of set size.

7 Models are compared to the best-fitting model from a factorial model comparison that used this

8 dataset (VP = variable precision, Poisson recall, with binding errors) (van den Berg et al., 2014).

9 Bayesian model selection favored a model that incorporated t-distributed memory reports, power-

10 law precision decrements and all modeled aspects of center-surround chunking $(\mathrm{C}+\mathrm{N}+\mathrm{T}+\mathrm{P})$. A model

11 lacking power-law precision decrements $(\mathrm{C}+\mathrm{N}+\mathrm{T})$ performed similarly in model comparison to the

12 VP model. B) Horizontal bars reflect AIC preference for the winning $(\mathrm{C}+\mathrm{N}+\mathrm{T}+\mathrm{P})$ model over the best

13 model that lacks chunking (VP) for each experiment and are arranged according to mean AIC

14 preference (with experiments providing strongest support for the more center-surround model on

15 top). C-G) Posterior predictive checks reveal nuanced discrepancies in the predictions across

16 models. Actual and simulated data were sorted by subject and set size and fit with a flexible mixture

17 model (see Methods) that estimated: guess rate (C), binding error rate (not shown), recall rate (D),

18 report precision (E), modulation of recall by chunking (F) and modulation of precision by chunking

19 (G). Points and lines reflect mean/SEM fits to subject data whereas lines/shading reflect mean/SEM

20 fits to simulated data for each model (models denoted by color: base = gray, $\mathrm{VP}=$ green, $\mathrm{C}+\mathrm{N}+\mathrm{T}=$

21 blue, $\mathrm{C}+\mathrm{N}+\mathrm{T}+\mathrm{P}=$ orange). All models captured guess and recall rates reasonably well (C\&D), but

22 only models that included either chunking $(\mathrm{C}+\mathrm{N}+\mathrm{T})$, precision decrements with set size (VP) or both

$23(\mathrm{C}+\mathrm{N}+\mathrm{T}+\mathrm{P})$ could account for changes in precision of reports across set size (E). Only models that

24 included chunking $(\mathrm{C}+\mathrm{N}+\mathrm{T} \& \mathrm{C}+\mathrm{N}+\mathrm{T}+\mathrm{P})$ could account for within set size modulation of recall (F).

25 Within set size modulation of precision was overestimated by a chunking model with fixed

26 assumptions about precision $(\mathrm{C}+\mathrm{N}+\mathrm{T})$ and underestimated by models without chunking (base \& VP)

27 but well estimated by a model that included chunking and allowed precision to vary with set size 
$1(\mathrm{C}+\mathrm{N}+\mathrm{T}+\mathrm{P}) . \mathrm{H})$ Bars indicate mean partitioning criterion for the $(\mathrm{C}+\mathrm{N}+\mathrm{T})$ model across the

2 experiments included in the meta-analysis (sorted from maximum). I) Correlation between mean

3 absolute error magnitude (z-scored per experiment and set size) and the best-fitting partitioning

4 criterion is plotted as a function of set size (abscissa). Points and lines reflect mean and bootstrapped

$595 \%$ confidence intervals, respectively.

8 Model comparison favored the most complex model, which incorporated all aspects

9 of chunking, flexible kurtosis of error distributions, and allowed precision to change

10 as a function of set size (exceedance probability $=0.96$, mean AIC relative to best

11 AIC $=4.7$; figure 10A). Performance of the model that included all aspects of

12 chunking and kurtosis but not precision decrements was similar to that of the VP

13 model (mean relative AIC 8.6 and 8.3 for $\mathrm{C}+\mathrm{N}+\mathrm{T}$ and VP models respectively; figure

14 10A). The advantage of the best performing chunking model was more prominent in

15 some studies than others. The studies using the widest range of set sizes showed the

16 largest chunking advantages (the Van den Berg et al. studies all included set sizes 1

17 through 8), whereas the study using the smallest range of set sizes (Gold et al, 2010;

183 or 4 items) showed a clear preference for the variable precision model over the

19 best chunking model. It is worthy of note that some of the experiments included in

20 the meta-analysis included additional manipulations or potential sources of

21 variability that might have been captured by the variable precision model but could

22 not possibly be accounted for in our chunking model, such as manipulation of the

23 duration of stimulus presentation, the duration of delay, and the day of a multi-day

24 experiment. Thus, despite the overall performance advantage of the best chunking 
1 model, it is still likely that some of the datasets include some residual variability that

2 could be captured by additional variability in precision across conditions.

3

4 In order to better understand why the most complex chunking model offered a

5 better fit to subject data, we performed a posterior predictive check by simulating

6 data from each model using the maximum likelihood parameter values and then

7 examining the simulated and actual data using an extended mixture model fit

8 separately to data for each set size (figure 10C-G). While all models captured the

9 general trends in recall and guessing (figure 10C-D), the base model was not capable

10 of fitting the changes in precision across set size observed in subject data, whereas

11 both the chunking models and variable precision model could capture these changes

12 (figure 10E). Only the models that included chunking were capable of accounting for

13 within-set size fluctuations in recall rate (figure 10F). Similarly, simulated data from

14 both chunking models produced within-set size modulations of precision that were

15 qualitatively similar to those observed in subjects, but the chunking model that

16 lacked the ability to modulate precision according to set size produced much larger

17 within-set size fluctuations in recall than were actually observed in the data. Thus,

18 the best-fitting chunking model improved on the VP model by capturing the effects

19 of center-surround chunking on recall and precision within set size. On the other

20 hand, the chunking model that did not allow precision to change with set size

21 seemed to capture set size precision effects by over-estimating the repulsive

22 interactions between items, leading to a worse fit than the more complex model that

23 allowed precision to change with set size. 
2 Across experiments and subjects, there were systematic differences in chunking that

3 related to overall performance. Mean partitioning criterion differed systematically

4 across experiments in a manner unrelated to overall model preference (figure $10 \mathrm{H}$ ).

5 While the number of experiments included in this analysis is small, it should be

6 noted that two of the experiments that included the least inclusive chunking

7 behaviors involved storing an orientation rather than a color. The other experiment

8 with a relatively small mean partitioning criterion (Wilken \& Ma 2004) used a color

9 sampling strategy that prevented fine grain estimation of the best-fitting

10 partitioning criterion: similar but non-identical colors were never included in a

11 single color array. Within experiments, subjects also differed in the partitioning

12 criterion that best described their behavior in a manner that related to performance.

13 Specifically, subjects that were best fit by the largest partitioning criterion values

14 also tended to make the smallest absolute errors in the high, but not low, set size

15 conditions (Figure 10I). Thus, individual differences in chunking may underlie

16 individual differences in performance for high memory load conditions that have

17 previously been thought to reflect differences in overall memory capacity.

\section{Discussion:}

22 Our work builds on two parallel lines of research. One has focused on how encoding

23 and decoding of working memories are optimized under various statistical 
1 contingencies (Brady et al., 2009; Brady \& Alvarez, 2011; Brady \& Tenenbaum,

2 2013; Lew \& Vul, 2015; Orhan \& Jacobs, 2013; Sims et al., 2012), whereas the other

3 has focused on understanding the nature of capacity limitations in visual working

4 memory (Bays et al., 2009; Bays \& Husain, 2008; van den Berg et al., 2012; 2014;

5 Zhang \& Luck, 2008; 2009; 2011). Here, we explore how people optimize encoding

6 in the same tasks that have formed the basis of our understanding of capacity

7 limitations. Our findings shed light on both the nature of memory capacity

8 limitations and on the encoding strategies employed to minimize their impact.

10 With regard to encoding strategies, the binary encoding model showed that

11 selective chunking allowed performance advantages for clustered stimulus arrays

12 that grew as a function of set size, could be learned according to trial feedback, and

13 limited asymptotic item storage to approximately four items (figure 3). Unlike

14 previous models that have examined how non-independent item encoding and

15 decoding schemes could affect memory performance (Brady et al., 2009; Brady \&

16 Alvarez, 2011; 2015; Lew \& Vul, 2015; Orhan \& Jacobs, 2013; Sims et al., 2012), our

17 model shows how memory storage could be optimized without foreknowledge of, or

18 even the existence of, statistical regularities in memoranda. Because of this, our

19 model provides unique insight into how subjects might optimize behavior in

20 standard working memory tasks, in which stimulus dependencies and

21 foreknowledge thereof are intentionally minimized. As predicted by our model,

22 human subjects display performance advantages when remembering clustered

23 stimulus arrays (figure $4 \& \mathrm{~S} 1$ ) that are not explained by binding errors (Figure 
14 D\&S2) and occur irrespective of whether the recalled item was itself in a stimulus

2 cluster (Figure S3). Furthermore, trial-to-trial adjustments in performance of

3 human subjects followed the same basic pattern through which chunking was

4 learned in our model; namely, rewarded employment of chunking on one trial

5 increased the probability of chunking on the next (Figure 4E\&F). Thus, our model

6 identifies and provides a normative explanation for a major source of performance

7 variability across trials in visual working memory tasks: selective chunking of

8 similar items into working memory and the optimization thereof.

10 Our findings are in line with previous work that highlights the use of chunking as a

11 mnemonic strategy in a wide range of working memory tasks (Cowan, 2001).

12 Chunking was first used to describe mnemonic strategies for storage of sequential

13 information, for example, encoding the digits 2-0-0-5 as a single date (2005) rather

14 than as its constituent digits (Chen \& Cowan, 2005; Cowan, 2001; G. A. Miller, 1956).

15 In the visual domain, visual features are in some sense chunked into objects (Luria \&

16 Vogel, 2014). Recent work has suggested that people can chunk arbitrary visual

17 information when that information is inherently clustered and visible for an

18 extended duration (Lew \& Vul, 2015). Here, we extend on this work to show that a

19 simple form of chunking, joint encoding of similar feature values, is rapidly

20 implemented by human visual working memory systems to improve performance in

21 tasks that have heretofore been thought to lack exploitable statistical structure. 
1 The basic computations necessary to achieve performance advantages through

2 chunking could be endowed to a recurrent neural network by implementing center-

3 surround dynamics (figure 5). These dynamics arbitrate a tradeoff between recall

4 and precision (figure 6) that was supported by empirical evidence of higher

5 precision representations for unclustered stimulus arrays (figure 7). This sort of

6 tradeoff between memory precision and item capacity is similar to that observed in

7 binding pool models of working memory, where the level of connectivity in the

8 binding pool controls a tradeoff between precision and quantity of representations

9 (Swan \& Wyble, 2014; Swan, Collins, \& Wyble, 2016). Here we show that this

10 tradeoff can be exploited to improve performance, and that human subjects seem to

11 do so. In particular, subjects demonstrated the performance benefits, response

12 biases, and costs in precision that were predicted by center-surround chunking and

13 were quantitatively best described by it (figures 5\&7-9). While the quantitative

14 advantage for the center-surround model was small (figure 9), this advantage was

15 likely limited in part by technical constraints that required simplification of the

16 center-surround model for fitting purposes, in particular by the removal of noise in

17 the initial color representation. Nonetheless, these findings are generally consistent

18 with previous work that has highlighted the effects of center-surround processing

19 on perception and memory (Almeida et al., 2015; Johnson, Spencer, Luck, \& Schöner,

20 2009; Störmer \& Alvarez, 2014; Xing \& Heeger, 2001). Furthermore, the specific

21 inter-item dependencies predicted by our model and observed in our empirical data

22 were consistent with those emerging from recurrent neural networks that rely on

23 tuned inhibition (Almeida et al., 2015), but not with those predicted by hierarchical 
1 models of memory decoding, as the latter do not produce repulsion of dissimilar

2 features (Brady \& Alvarez, 2015; Orhan \& Jacobs, 2013).

3

4 Our center-surround model serves not only to describe nuanced features of

5 behavior, but also to link our findings to potential biological mechanisms. We show

6 that a small change to a prominent neural network model of working memory

7 maintenance, namely the incorporation of tuned inhibition, provides the model with

8 the capability to chunk similar features into a joint representation but partition

9 dissimilar ones through repulsion (figure 5; (Ben-Yishai et al., 1995; Kohonen, 1982;

10 Murray et al., 2014; Somers et al., 1995; X. J. Wang, 1999; Wei et al., 2012)). Our

11 descriptive model based on this mechanistic account is supported by the frequent

12 observation of sustained activity during the delay period of memory tasks in both

13 parietal and prefrontal cortices (Funahashi, Bruce, \& Goldman-Rakic, 1989; Fuster \&

14 Alexander, 1971; Gottlieb, 2004) (but see also (Lara \& Wallis, 2014)). Here we have

15 considered the network to store features on a single dimension (color); however, it

16 is clear that at some level, conjunctive coding across features (e.g. color and

17 orientation) is necessary to bind information to the dimension used to probe

18 memories (Matthey, Bays, \& Dayan, 2015). In our task, it is unknown whether any

19 sustained representations reflect information about the report feature (color in our

20 task), probe feature (orientation in our task), or some conjunction of the two. Recent

21 work has hinted that in some cases, sustained representations in prefrontal cortex

22 may only encode the probe dimension, which could point back to relevant sensory

23 representations at time of recall (Ester, Sprague, \& Serences, 2015; Kriete, Noelle, 
1 Cohen, \& O'Reilly, 2013; Lara \& Wallis, 2014; 2015). Previous computational

2 instantiations of this process have relied on the basal ganglia to learn appropriate

3 prefrontal representations that can be jointly cued by multiple disparate perceptual

4 features, based on reward feedback (A. G. E. Collins \& Frank, 2013; Frank \& Badre,

5 2012; Kriete et al., 2013). Analogously, feedback effects observed in our data could

6 be driven by the basal ganglia learning to selectively engage prefrontal units that are

7 prone to representation of multiple probe feature values. This interpretation could

8 expand on a large body of work that implicates the basal ganglia in gating working

9 memory processes by stipulating a novel and testable role for the basal ganglia in

10 optimizing joint feature encoding (Chatham, Frank, \& Badre, 2014; A. G. E. Collins \&

11 Frank, 2013; Hazy, Frank, \& O’Reilly, 2006; O'Reilly \& Frank, 2006; Voytek \& Knight,

12 2010).

13

14 An important question stemming from our work is to what extent chunking can be

15 adjusted to optimize working memory accuracy under different conditions. Our

16 modeling shows that a simple learning rule is capable of rapidly adjusting the

17 amount of chunking to optimize performance given the current memory demands,

18 leading to greater chunking for higher memory loads. Individual differences in

19 chunking were selectively related to performance in the highest memory load

20 conditions (figure 9E \& 10I); however, neither our experiment, nor the meta-

21 analytic dataset explicitly manipulated chunking incentives over a time-course long

22 enough to measure learning effects. Nonetheless, even in the absence of explicit

23 manipulation, feedback-dependent modulation of chunking behaviors in our 
1 experimental data was indicative of online optimization of the chunking process

2 (figure 4D-F), such as the process that allowed learning of the partitioning criterion

3 in the binary encoding model (figure 4G). Yet these trial-to-trial adjustments occur

4 despite only minimal performance improvements across task blocks (figure S2).

5 There are several possible explanations for this discrepancy, including 1) that a

6 priori processing strategies are well-calibrated to our task, 2) that optimization in

7 our task occurs on a different timescale than our measurements, and 3) that the

8 presence of uniformly spaced arrays hindered learning overall. Distinguishing

9 between these possibilities will require a better understanding of what exactly is

10 being adjusted in response to feedback. For example, reward feedback could

11 promote the prioritization of storing chunked arrays over non-clustered ones, or it

12 could modulate center-surround inhibition dynamics (e.g., via fine tuning of feature

13 selective attention and/or altering local excitation-inhibition balance (Störmer \&

14 Alvarez, 2014; Wei et al., 2012)). In any case, our work, along with other recent

15 research showing an adaptive tradeoff of precision and recall (Fougnie, Cormiea,

16 Kanabar, \& Alvarez, 2016), strongly motivate future work to better understand the

17 scope, time course, and mechanism for this type of optimization process.

20 Implications for capacity limitations. Working memory limitations have been

21 theorized to result from either a discrete limitation on available "slots" or a

22 continuous limitation by a divisible "resource". The distinction between these

23 theories is most evident when additional targets are added to a memory array. A 
1 discrete limitation predicts that after all slots are filled, additional targets will be

2 forgotten and will be reported as random guesses (Luck \& Vogel, 2013). In contrast,

3 a resource limitation predicts that additional targets will cause each target to be

4 encoded with lower precision (Ma et al., 2014). While individual studies have

5 provided support for each theory (Bays et al., 2009; Bays \& Husain, 2008; Cowan \&

6 Rouder, 2009; Chris Donkin et al., 2013a; Christopher Donkin et al., 2013b; Pratte,

7 Park, Rademaker, \& Tong, 2017; Rouder et al., 2008; van den Berg et al., 2012;

8 Zhang \& Luck, 2008; 2009; 2011), a recent meta-analysis provides simultaneous

9 support for the core predictions of both: increasing memory load leads to both

10 increased guessing and decreased precision (van den Berg et al., 2014).

12 Our results suggest that a joint capacity limitation over recall and precision may

13 result in part from a rational chunking procedure implemented through center-

14 surround dynamics to effectively trade precision for recall (figure $5 \& 6$ ). This

15 procedure allows subjects to achieve performance improvements for clustered

16 stimulus arrays at the cost of precision (figures 6). It is also capable of explaining

17 decrements in precision with set size, as larger sets of items lead to increased

18 repulsive forces experienced by each individual item (figure 10E). In addition to

19 accounting for known influences on precision, our model also predicted that

20 measured precision should vary across trials, an established feature of human

21 behavioral data (Fougnie et al., 2012; van den Berg et al., 2012), and correctly

22 predicted that this variability in precision should depend on the features of non-

23 probed targets (figure 8D\&E; 10G). Nonetheless, the best-fitting center-surround 
1 chunking model employed leptokurtic memory reports in order to capture

2 additional variability in precision that was not accounted for by the chunking and

3 repulsion processes alone, suggesting that other factors must also contribute to

4 variability in precision (figure 10A). Furthermore, the best-fitting model also

5 allowed memory report precision to vary as a power function of set size, as this

6 appropriately balanced the magnitude of across set size (figure 10E) and within set

7 size (figure 10G) precision effects. Thus, center-surround chunking, as we

8 implemented it, can quantitatively account for most, but not all, of the changes in

9 precision across trials and set-sizes.

11 Our findings also call the interpretation of precision measurements into question.

12 The center-surround model predicts that internal representations apply attractive

13 and repulsive forces to one another, systematically biasing memory reports. When

14 averaged across trials with differing stimulus configurations, such interactions are

15 interpreted as variability in memory reports, as the net forces on a probed target

16 vary randomly from one stimulus configuration to the next. Yet, since much of this

17 variability is simply an artifact of averaging across disparate conditions, our work

18 raises an important question: how much of the variability in memory reports across

19 trials and individuals is truly reflective of imprecision, rather than bias? While the

20 notion that imprecision can emerge from systematic inter-item dependencies is

21 somewhat at odds with the basic resource limitation model, it is consistent with the

22 recent proposal of a specific form of resource limitation in which the constrained 
1 resource is the representational space itself (M. A. Cohen, Rhee, \& Alvarez, 2016;

2 Franconeri, Alvarez, \& Cavanagh, 2013; Oberauer \& Lin, 2017).

3

4 Within such a framework, it is interesting to reconsider the meaning of individual

5 differences in memory storage recall and precision. Previous work has shown that

6 individual differences in the number of items successfully retained in visual working

7 memory tasks, but not differences in precision, are related to fluid intelligence and

8 psychiatric conditions such as schizophrenia, among other factors (Fukuda, Vogel,

9 Mayr, \& Awh, 2010; James M Gold et al., 2010). These results have been interpreted

10 in terms of differences in a discrete capacity for memory storage, or in filtering

11 irrelevant information (Vogel, McCollough, \& Machizawa, 2005), but our results

12 suggest that some of these individual differences may be driven instead by

13 differences in chunking behavior or the optimization thereof. To this effect, we

14 showed both in our own dataset and in the meta-analytic dataset that individual

15 differences in task performance, particularly at high set sizes, were systematically

16 related to differences in chunking policy, with subjects that chunked most liberally

17 achieving the best performance for higher set sizes (figures 9E\&10I). Given that the

18 best chunking policies in our binary encoding model for a set size of five were quite

19 liberal (figure 3A,C,D,F) we suspect that a number of subjects could have improved

20 performance dramatically, were they to have chunked more liberally. In fact, a

21 subset of subjects was best fit by models that fully partitioned color information

22 (figure 9A), achieved lower overall performance (figure 9E), and likely attenuated

23 the aggregate performance advantages for clustered stimulus arrays (compare 
1 figure 3L to figure 4A). It is not clear to what extent these individual differences in

2 chunking policy result from differences in the ability to learn an appropriate

3 criterion, or from hard-wired differences that might predispose individuals toward

4 either chunking or partitioning neural representations. Our neural network model

5 suggests that performance-based measures of capacity may be sensitive to

6 individual differences in lateral connectivity profiles that favor a spectrum from

7 independent to merged feature storage policies, and to the ability to override such

8 policies through learned top-down modulation of lateral connectivity (Freeman,

9 Driver, Sagi, \& Zhaoping, 2003; Freeman, Sagi, \& Driver, 2001; Lowe et al., 2016).

11 In summary, our results show that humans readily exploit chunking strategies to

12 improve performance on visual working memory tasks. The implementation of

13 chunking is consistent with a form of center-surround dynamics that combines

14 similar representations and facilitates mutual repulsion of disparate ones. This

15 implementation leads to a fundamental tradeoff between the number of items

16 stored and the precision with which they are stored, providing a natural bridge

17 between slots and resource accounts of working memory capacity limitations.

18 People optimize this tradeoff from trial-to-trial according to stimulus statistics and

19 evaluative feedback in a manner that differs across individuals and is predictive of

20 working memory task performance. These results provide a normative joint account

21 of how and why discrete and continuous factors contribute to working memory

22 capacity limits across individuals and task conditions. 
Methods:

4 Delayed report task. 54 human subjects completed five blocks (100 trials each) of a

5 delayed report color reproduction task (figure 2). Each trial of the task consisted of

6 four primary stages: stimulus presentation, delay, probe, and feedback. During

$7 \quad$ stimulus presentation, subjects were shown five oriented bars (length $=2$ degrees

8 visual angle) arranged in a circle (radius $=4$ degrees visual angle) centered on a

9 fixation point. Bar positions were equally spaced around the circle and jittered

10 uniformly from trial to trial. Bar orientations were uniformly spaced, jittered from

11 trial to trial, and independent of position or color. Bar colors were chosen from a

12 fixed set of colors corresponding to a circle in CIELAB color space ( $L=80$, radius in

$13 \mathrm{a}^{*}, \mathrm{~b}^{*}=60$ ) and referred to by angular position for convenience. In the "random

14 spacing" condition, all five colors were sampled independently of one another from

15 the color space, allowing for the possibility of two similar colors in the same

16 stimulus array. In the "fixed spacing" condition, colors were uniformly spaced along

17 the CIELAB color wheel and randomly assigned to bar locations. Stimuli were

18 presented for $200 \mathrm{~ms}$, after which the screen was blanked.

20 The subsequent delay period lasted $900 \mathrm{~ms}$, during which subjects were forced to

21 remember the colors and orientations of the preceding stimulus array. During the

22 subsequent probe stage, subjects were shown a gray oriented bar in the center of

23 the screen for one second, before being asked to report the color that had been 
1 associated with that orientation in the preceding stimulus array. Color reports were

2 made by adjusting the color of the oriented bar using a mouse. The initial position of

3 the mouse on the color wheel was randomly initialized on each trial. On a subset

$4(1 / 3)$ of trials, subjects were asked to make a post-decision wager about the

5 accuracy of their report by choosing to bet either 0 or 2 points. Binary feedback was

6 provided on each trial based on whether subject reporting accuracy fell within a

7 certain error tolerance window ( $\pi / 3$ radians - low precision condition $[26$

8 subjects] or $\pi$ /8 radians - high precision condition [28 subjects]). A priori target

9 sample size for each group was set to twenty-four based on other studies in the field

10 (without explicit power calculations). Additional subjects were recruited beyond

11 this to account for potentially unusable data (e.g. subjects guessing on all trials).

12 Four subjects in the high precision condition and three subjects in the low precision

13 condition were removed from analyses because of error distributions that were not

14 statistically distinguishable from uniform guessing (error variance $>0.91$ ), leading

15 to sample sizes of 23 and 24 for low and high precision conditions respectively. All

16 subjects were paid bonuses according to total accumulated points. All human

17 subject procedures were approved by the Brown University Institutional Review

18 Board and conducted in agreement with the Declaration of Helsinki.

20 Binary encoding model. To explore the potential advantage of chunking in delayed

21 report tasks, we developed a flexible and computationally tractable model for

22 capacity-limited storage. This model stores color and orientation information

23 symbolically in a set of binary "words" concatenated to form a "sentence". During 
1 the stimulus presentation phase, target colors and orientations are "encoded" as an

2 alternating sequence of binary words reflecting the position on a circular feature

3 space (figure 3). The number of binary digits (bits) in a word controls the precision

4 with which the feature is stored. For example, a single digit can encode which half of

5 the feature space contains the color of a bar, whereas three bits can narrow the

6 stimulus color down to one eighth of the color space (figure 3, top). Each binary

7 word is followed by a "stop" symbol denoting the type of information in the

8 preceding word (e.g. color or orientation). A capacity limitation is implemented in

9 the model as a limit on the number of bits that can be stored in memory. Specifically,

10 we applied a fixed limit of 15 bits for storage of color information. Similar results

11 were achieved by applying a limit to the total bits, i.e. including orientation

12 information, but here we allow for perfect orientation storage in order to isolate the

13 effects of capacity limitations on the recall dimension (color).

15 Bits were allocated in two different ways: in one set of simulations, bits were

16 assumed to be continuously divisible (analogous to resource models) and in the

17 other set of simulations, bits were not divisible beyond binary units (analogous to

18 slots-and-averaging models). For resource model simulations, performance was

19 computed analytically according the following error function:

20

$$
\text { Error }=\left|\Delta \mu+\int_{r=0}^{R}\left(\frac{R}{2}-r\right) d r\right|
$$


1 where $\Delta \mu$ is the difference in the "chunk" mean and the true color and $\mathrm{R}$ is the

2 continuous range over possible stimulus values specified by the encoding model,

3 which depends on the number of bits allocated to each item according to the

4 following function:

5

$$
R=\frac{2 \pi}{2^{b}}
$$

7 where $b$ is the number of bits allocated to each target, which in turn depends on the

8 total number of items and the exact pattern of chunking and partitioning across the

9 stimulus values. Chunking and partitioning were controlled in three ways: 1) in the

10 fully partitioning model, all colors were represented separately, 2) in the optimal

11 partitioning model, all possible partitioning patterns were considered for each

12 stimulus array and only the performance of the best partitioning pattern was

13 reported, and 3) in the criterion-based partitioning model, adjacent colors were

14 partitioned (represented distinctly) if they were separated by a distance that

15 exceeded the partitioning criterion. Colors that were not separated by partitions

16 were combined into a single "chunk" and represented by their mean value. This

17 procedure could sometimes lead two colors that were separated by distances

18 greater than the partitioning criterion to be included in the same chunk, if they were

19 both sufficiently close to an intermediate color. The performance of the criterion-

20 based partitioning model was computed across a range of possible partitioning

21 criterions and the performance of the best-performing criterion across all trials for a

22 given set size was reported (see figure $4 \mathrm{~A}$ ). 
2 In the second set of simulations where bits were considered to be indivisible,

3 analogous to the slots + averaging framework, model performance was assessed

4 through exhaustive simulations. In this framework, bits were as evenly distributed

5 among represented colors as was possible for a given stimulus array, as this

6 strategy for allocation of bits achieved the best performance. During the probe

7 phase, the model is presented with a single orientation and recalls the color word

8 that immediately precedes that orientation in the stored binary sentence. A report is

9 then sampled from a uniform distribution across the range of colors consistent with

10 that stored binary color word. For example, if the color word contains one, two, or

11 three bits, it is sampled from uniform distribution over one half, quarter, or eighth

12 of the color space.

13

14 Chunking was parametrically implemented in the binary encoding model by adding

15 a "partitioning criterion" that specifies the minimum distance between two colors in

16 color space that is necessary for independent storage. Colors separated by distances

17 greater than the partitioning criterion are partitioned, and all colors that are not

18 separated by a partition are combined into a single color representation. The

19 distance computation is completed during the "encoding" phase, before colors are

20 converted to binary words. Distances are corrupted with a small amount of

21 independent noise consistent with variability in the visual representation or the

22 chunking processes (normally distributed with standard deviation equal to 0.4

23 times the partitioning criterion). This noise gave rise to variability in the chunking 
1 process, such that a given set of stimuli might be partitioned differently on different

2 trials. After chunking, bits are allocated evenly across all represented colors, as

3 described above.

5 Model performance was simulated for the delayed estimation task across eight

6 different array sizes (1-8) with two different color generation conditions (fixed- and

7 random-spacing) for nine different partitioning criterions ranging from zero to $\pi$.

8 For each condition and model, mean absolute error was computed across 5000

9 simulated trials. The best chunking model (see figure $4 \mathrm{~L}$ ) was defined as the model

10 with the lowest mean absolute error, whereas the fully partitioned model was the

11 model with partitioning criterion equal to zero (such that every color was stored

12 independently). For each condition, chunking bonus was computed as the difference

13 in absolute error between the non-chunking and best-chunking models.

15 For the trial-to-trial optimization of the partitioning criterion (figure 4g), we

16 adjusted the partitioning criterion on each trial according to the following rule:

$$
P C=P C-\alpha \delta \Delta C
$$

19 where $\mathrm{PC}$ is the partitioning criterion, $\alpha$ is a learning rate, $\delta$ is a reward prediction

20 error (previous trial feedback minus long term average feedback), and $\Delta C$ is the

21 number of "chunks" into which the previous stimulus array was divided minus the

22 long term average of that quantity. Thus, if by chance the model did more chunking 
1 on a given trial, the $\Delta C$ would take a negative value, and positive feedback would

2 drive a positive $\delta$ and a corresponding increase in the partitioning criterion, leading

3 to an increase in chunking on subsequent trials. Negative feedback for the same trial

4 would lead to a negative $\delta$ and corresponding decrease in the partitioning criterion,

5 leading to a decrease in chunking on subsequent trials.

$7 \quad$ Computing array clustering. In order to assess the potential benefits of chunking on

8 each trial we computed a clustering statistic, within-cluster variance (WCV), for

9 each stimulus array. WCV was computed by dividing the array colors into two

10 clusters that minimized the mean variance within the clusters. WCV was defined as

11 the average circular variance over colors within these clusters.

13 Logistic regression models. Binary accuracy and betting data were concatenated

14 across all subjects and interrogated with a mixed-effects logistic regression model

15 that included terms to account for fixed effects of 1$)-\log (\mathrm{WCV})$, a proxy for stimulus

16 array chunkability, 2) the color distance between the probed target and each other

17 color in the array, ordered from smallest to largest, 3) feedback on previous and

18 subsequent trials, 4) spatial distance between the location of the probed target and

19 the location of the previously probed target, and 5) task block. In addition, the

20 model included dummy variables to account for random intercepts specific to

21 individual subjects. The same analysis was applied to data simulated from the best-

22 fitting mixture model, which considered all reports to come from a weighted

23 mixture of recall, uniform guess, and binding error distributions (Bays et al., 2009). 
2 Mixture model. We extended the standard mixture model of memory reports (Bays

3 et al., 2009; Zhang \& Luck, 2008) to allow for modulation of recall probability,

4 precision, and bias according to WCV, nearest neighbor distance, and feedback. The

5 standard mixture model assumes reports are generated from a mixture of "correct

6 recall", "guessing", and "binding error" processes. These three mixture components

7 were specified using two free parameters: one dictating the probability with which

8 an item would be successfully stored (correct recall + binding error) and one

9 specifying the probability with which a stored item would be correctly reported

10 (correct recall+binding error $)$. We allowed the parameter dictating successful storage

11 to be modified as a logistic function of 1) $\log (\mathrm{WCV}), 2$ ) previous feedback, 3)

12 previous $\log (\mathrm{WCV}), 4)$ previous feedback* ${ }^{*} \log (\mathrm{WCV})$ and 5) previous

13 feedback*log(WCV)*previous $\log (\mathrm{WCV})$. All potential modulators of successful

14 storage were mean-centered (before and after interaction) and constrained by

15 priors favoring values near zero $[\sim \operatorname{normal}(0,0.5)]$. Since our successful storage

16 parameter is the probability the subject will not elicit a uniform guess, it affects both

17 correct recall and binding error mixture components. However, since reports were

18 far more likely to correspond to correct recalls (median mixture proportion $=0.50$

19 across subjects) than binding errors (median binding error proportion $=0.17$ across

20 subjects), modulator coefficients had larger effects on recall than binding errors,

21 and we refer to them in the results as modulating recall for simplicity. We also

22 considered an alternative model in which modulators affected the recall term

23 directly and found similar results, although this alternative model provided a worse 
1 overall fit of the data. Group mean parameter estimates were tested against the null

2 hypothesis (estimate $=0$ ) with a classical one sample t-test; however, in cases where

3 moderate $p$ values were observed $(p<0.1 \& p>0.01)$ Scaled-Information Bayes

4 factors were computed to quantify the model evidence in favor of the alternative

5 hypothesis relative to that of the null hypothesis (rscale=0.707) \{Rouder:2009ij\}.

$7 \quad$ Neural network simulations. Neural network simulations were conducted using a

8 basic recurrent neural network that has been described previously (Wei et al.,

9 2012). The model consists of 2048 pyramidal (excitatory) neurons and 512

10 inhibitory interneurons. Pyramidal neurons had the following cellular properties:

$11 \mathrm{C}_{\mathrm{m}}=0.5 \mathrm{nF}, \mathrm{g}_{\text {leak }}=0.025 \mu \mathrm{S}, \mathrm{V}_{\text {leak }}=-70 \mathrm{mV}, \mathrm{V}_{\text {thresh }}=-50 \mathrm{mV}, \mathrm{V}_{\text {res }}=-60 \mathrm{mV}, \tau=1 \mathrm{~ms}$.

12 Interneurons had the following cellular properties: $\mathrm{C}_{\mathrm{m}}=0.2 \mathrm{nF}, \mathrm{g}_{\text {leak }}=0.02 \mu \mathrm{S}, \mathrm{V}_{\text {leak }}=$

$13-70 \mathrm{mV}, \mathrm{V}_{\text {thresh }}=-50 \mathrm{mV}, \mathrm{V}_{\text {res }_{-}}=-60 \mathrm{mV}, \tau=1 \mathrm{~ms}$. The model included AMPA, NMDA,

14 and GABA receptors with properties described previously (Furman \& Wang, 2008).

15 Pyramidal-to-pyramidal connection weights followed a narrowly tuned Gaussian

16 profile across stimulus space $\left(\sigma=5, \mathrm{~J}^{+}=5.6\right)$. Pyramidal-to-interneuron and

17 interneuron-to-pyramidal connectivity profiles were identical, and in one set of

18 simulations fully connected with uniform weights (figure 5C). In the second set of

19 simulations, the cross-population connectivity was defined by a mixture of uniform

20 weights and broadly tuned Gaussian weights $(\sigma=20$, mixture proportion $=0.1)$.

21 Input was delivered to both networks for $200 \mathrm{~ms}$ through activation of an AMPA

22 current with $g_{\max }=0.57$ using a spatial profile that was centered on 5 "target colors"

23 with a Gaussian profile $(\sigma=4)$. Stimulus delivery was followed by a delay period 
1 during which no input was provided to the network and activity was sustained

2 completely through recurrent connectivity.

3

4 Center-surround chunking model. To determine the effects that center-surround

5 dynamics would have on visual working memory task performance, we extended

6 the standard descriptive model of delayed memory reports to incorporate features

7 of center-surround dynamics. In particular, on each trial, internal representations of

8 each color were generated from a von Mises distribution with fixed concentration (7

9 for simulations). Pairwise distances (in color space) were computed for each pair of

10 internal representations. Chunking probability was computed as a scaled von Mises

11 function of this distance $(\mu=0, \kappa=12$ for simulation), corresponding to the narrow

12 excitatory "center" over which local representations are likely to attract one another

13 (figure 5A-C). Representations were merged in accordance with these chunking

14 probabilities by replacing the color associated with each merged representation

15 with the mean of the merged colors. After probabilistic chunking, distances were

16 recomputed between representations, and each representation applied a repulsive

17 force on neighboring representations as defined by a scaled von Mises function of

18 the re-computed distance $(\mu=0, \kappa=2$ for simulation), corresponding to the broadly

19 tuned "surround" over which representations repulse one another (figure 5A-C).

20 Applying these forces leads each representation to be reset according to the

21 following equation: 


$$
\operatorname{color}_{i} \leftarrow \text { color }_{i}+\sum_{X \neq i} W_{\text {surround }}\left(\frac{e^{\kappa \cos \left(\operatorname{color}_{i}-\text { color }_{x}\right)}}{2 \pi \mathrm{I}_{0}(k)}\right)\left(\frac{\mid \operatorname{color}_{i}-\text { color }_{x} \mid}{\operatorname{color}_{i}-\operatorname{color}_{x}}\right)
$$

2 where $W_{\text {surround }}$ is a weight that controls the overall magnitude of surround effects,

3 the second term in the sum is the probability density function for a von Mises

4 distribution, and the final term serves to ensure that targets exert repulsive forces

5 on neighboring targets. For the simulations in figure 5, the weight parameters for

6 both center and surround were set to equal values ranging from 0 to 0.7 . For

7 comparisons to subject data, $W_{\text {surround }}$ was set to 0.6 and $W_{\text {center }}$ was set to 1.2 .

9 Probabilistic recall was implemented in the model according to a Poisson memory

10 process (Sims et al., 2012; van den Berg et al., 2014). On each trial, the model

11 accurately recalled some number of representations drawn from a Poisson

12 distribution ( $\lambda=2$ for simulations). Similar results were achieved using an

13 inhibition based forgetting process inspired by Wei and colleagues (Wei et al.,

14 2012); however, here we use a more standard Poisson process for simplicity. In the

15 case that a representation that was not successfully recalled was probed, the model

16 reported either a uniformly distributed guess $(p=0.65)$ or the color of an

17 alternative representation (binding error, $\mathrm{p}=0.35$ ).

19 Quantitative model fitting. In order to estimate model-likelihood directly, the center-

20 surround chunking model was modified to allow for a closed-form likelihood

21 function. To this end, we stipulated that internal representations would perfectly 
1 reflect the true stimulus colors before being subjected to chunking and repulsion

2 processes instead of assuming that internal representations were subject to

3 variability resulting from perceptual processing (as described above). In order to

4 improve gradient descent, we implemented chunking using a gamma distribution

5 over partitioning criterions in which the mean of the distribution was fit as a free

6 parameter and the variance was fixed to 0.01 . The repulsion process was simplified

7 to a linear function of inter-item similarity, with a slope that was fit as a free

8 parameter and could take either positive values to capture attraction or negative

9 values to capture repulsion. Three versions of the simplified chunking model were

10 fit to delayed report data: 1) a center-only model in which the partitioning criterion

11 mean was fit as a free parameter and the repulsion coefficient was fixed to zero, 2) a

12 surround-only model in which the partitioning criterion mean was fixed to zero and

13 the repulsion coefficient was fit as a free parameter and 3) a center-surround model

14 in which both terms were fit as free parameters. In addition, all models included the

15 following free parameters: 1) Poisson lambda to describe the number of items that

16 would be stored on a given trial, 2) binding error fraction to describe the frequency

17 that reports would be generated from a non-probed representation, and 3)

18 precision of the report distribution. All models were compared to a basic mixture

19 model (Bays et al., 2009) using to penalize for complexity, as AIC allowed for better

20 model recovery from simulated data than did BIC. AIC values are reported for each

21 model relative to the lowest AIC model achieved by any model for a given subject

22 (relative AIC). Bayesian model selection was performed using -1/2 AIC as a proxy 
1 for model evidence with the SPM toolbox (Stephan, Penny, Daunizeau, Moran, \&

2 Friston, 2009).

3

4 Meta-analysis. In order to test the robustness of our findings and determine how the

5 behavioral hallmarks of chunking scale with the size of the stimulus array, we

6 applied a modified version of our mixture model to a meta-analysis dataset. The

7 meta-analysis dataset included eight studies and a total of 101 subjects (Bays et al.,

8 2009; James M Gold et al., 2010; Rademaker, Tredway, \& Tong, 2012; van den Berg

9 et al., 2012; Wilken \& Ma, 2004; Zhang \& Luck, 2008). Seven of the datasets,

10 available online at http://www.cns.nyu.edu/malab/resources.html, were originally

11 compiled by van den Berg et al. and have previously been described in detail (van

12 den Berg et al., 2014). Three of the studies compiled by Van den Berg et al. were

13 excluded from our analyses due to retraction of the original studies, although the

14 inclusion of these studies did not qualitatively change our results. The eighth

15 dataset (28 subjects) comprised the control subjects in a psychiatric comparative

16 study of visual working memory (James M Gold et al., 2010). Each study differed in

17 experimental details but involved a delayed report working memory task with at

18 least two different array sizes.

20 Quantitative model fitting to meta-analytic data. We constructed a nested set of

21 models to better understand whether chunking could improve explanations of

22 behavior in previous studies visual working memory studies. Each model was

23 extended beyond a "base" model in which the partitioning criterion was fixed to 
1 zero. The base model included one change from the models that were fit to the data

2 from our experiment in order to account for the possibility that binding errors

3 depend on set size (which was variable in the meta-analytic data but fixed in our

4 own study). Specifically, we replaced the fixed-probability of binding errors with a

5 Poisson distribution that described the number of probe dimension features that

6 would be recalled on a given trial $(s)$, with lambda of this distribution fit as a free

7 parameter. For each trial, this distribution was used to compute a probability that

8 the relevant probe feature would not be stored in memory:

9

$$
p(\text { forgot probe })=1-\sum_{s=0}^{\infty} p(s) * \min \left(1, \frac{s}{n}\right)
$$

11 where $n$ is the number of targets presented on a given trial and $\mathrm{p}(s)$ is the

12 probability of recalling $s$ probe dimension features on a Poisson distribution. The

13 minimum term accounts for the case where the number of available items is smaller

14 than the number of probe dimension features that could have been stored on a given

15 trial.

16

17 The probability of making a binding error, given that the recall feature was

18 remembered, was then computed as:

19

$$
p(B E)=p(\text { forgot probe })-\frac{p(\text { forgot probe })}{n}
$$


1 to correct for the possibility that, in the case that the model did not correctly store

2 the probe dimension feature, it could choose the correct report dimension feature

3 by chance. This change allowed the model to capture tendencies for binding errors

4 to increase with set size, as have been reported previously (Bays et al., 2009).

6 The first extension to the base model allowed the partitioning criterion and

7 repulsion criterion to be fit as free parameters, rather than set to zero as they were

8 in the base model. This extension allowed the model to capture the biases and recall

9 benefits that are predicted by our more general center-surround chunking model

10 (e.g. figures 5-8); however, it would not capture variability in reports that would be

11 expected to occur through the amplification of sensory noise by the chunking and

12 repulsion processes, as the sensory noise was removed in order to allow for a closed

13 form likelihood function.

15 In order to account for the basic effects of chunking and repulsion on report

16 variability that would be expected based on our center-surround chunking model,

17 but maintain the tractability of our likelihood function, we added two additional

18 parameters to the model to allow the variance in memory reports to scale linearly

19 with 1) the variance of feature values stored within a single chunk [chunking noise],

20 and 2) the total repulsive forces experienced by the recalled feature [repulsion

21 noise]. 
1 We also considered an extension that employed a more flexible report distribution

2 that included an additional free parameter to model differences in kurtosis. In this

3 extension, memory reports were generated from a t-distribution centered on the

4 value of the internal representation and truncated at that value plus or minus $\pi$

5 radians. The t-distribution included a base scale parameter fit to each subject, which

6 accounted for overall variability in memory reports and was incremented by the

7 additional chunking and repulsion variability as described above. In addition, the t-

8 distribution included a degrees of freedom parameter that was fit to each individual

9 subject, which allowed the model to capture report distributions ranging from

10 leptokurtic (low degrees of freedom) to mesokurtic (high degrees of freedom).

11

12 Finally, we considered an extension to the model that included the possibility that

13 precision depends on set size. Specifically, we stipulated that precision, or inverse

14 variance, of memory reports would obey a power-law relationship with set size:

$$
\frac{1}{\sigma_{\text {report }}^{2}}=J * n^{\alpha}
$$

17 where $J$ is the response precision expected when set size is equal to 1 , and $\alpha$ is the

18 power delineating the dependency of precision on set size, with negative values of $\alpha$

19 corresponding to precision values that decay with set size. 
1 The nested set of models were tested against one another, and also compared to a

2 variable precision model that includes Poisson item limits and binding errors that

3 scale linearly with set size, which was the best performing model in a previous

4 meta-analysis of delayed report working memory behavior (van den Berg et al.,

5 2014). Model comparison using AIC and Bayesian model selection was done as

6 described above. Posterior predictive checks were conducted by fitting actual and

7 model-generated meta-analytic data with a descriptive model of memory report

8 distributions separately for each subject and set size. The descriptive model

9 estimated the rate of three response types (guess, binding errors, and correct recall)

10 and the precision of memory reports as has been described previously (Bays et al.,

11 2009). However, the model also included two additional terms to capture

12 fluctuations in recall and precision within a given set size that would be predicted

13 by chunking. Specifically, the model allowed the probability of recall to vary as a

14 logistic function of trial-to-trial recall probabilities extracted from the center-

15 surround chunking model, and allowed the variance of the report distribution to

16 vary as a linear function of the trial-to-trial prediction for response variance

17 extracted from the center-surround chunking model. Trial-to-trial model

18 predictions were extracted from the center-surround chunking model that included

19 chunking and repulsion noise as well as t-distributed errors, as this model provided

20 a combination of a good fit to most subject data and relatively well-behaved

21 parameter estimates. 
1 Nearest neighbor analysis. For each trial, the nearest neighbor color was identified as

2 the color of the non-probed target that was most similar to that of the probed target.

3 Target colors and subject reports were transformed for each trial such that the

4 probed target color corresponded to zero and the nearest neighbor color ranged

5 from zero to $\pi$. Trials were then sorted according to absolute nearest neighbor

6 distance (see figure S2) and binned in sliding windows of 50 trials according to

7 nearest neighbor distance. Binned data were combined across all subjects and fit

8 with a mixture model that assumed data were generated from a mixture of 1) a von

9 Mises distributed memory report (free parameters: mean, precision, and mixture

10 weight), 2) uniformly distributed guesses (free parameters: mixture weight), and 3)

11 binding errors that were von Mises distributed and centered on non-probed targets

12 (no free parameters required, as mixture weight forms simplex with the other

13 mixture components). Maximum posterior probability parameter estimates for the

14 mixture model fits to subject and model simulated data are reported in figure 9

15 (prior distributions for all modulator terms were centered on zero with $\sigma=0.5$ for

16 recall modulators, $\sigma=2$ for precision modulators, and $\sigma=0.05$ for bias modulators).

\section{Author Note:}

19 We thank Ronald van den Berg, Wei Ji Ma and Jim Gold for sharing data from

20 previously published delayed report studies. We thank Xiao-Jing Wang for providing

21 code for neural network simulations. We thank Karen Schloss for help with accurate

22 color rendering. We thank Anish Aitharaju, Anthony Jang, Ji Sun Kim, Michelle

23 Kulowski, and Ezra Nelson for aiding in data collection. We thank Nicholas Franklin 
1 and Nathan Vierling-Claassen for helpful discussion. This work was funded by NIMH

2 grant F32 MH102009 and NIA grant K99AG054732 (MRN), as well as NIMH grant

3 R01 MH080066-01 and NSF grant \#1460604 (MJF). A previous version of this work

4 was presented at the New England Research on Decision Making (NERD) conference

5 and was published as a preprint on biorxiv:

6 http://biorxiv.org/content/early/2017/01/06/098939.

\section{Competing interests:}

9 The authors declare no competing interests.

\section{References:}

13

14 Almeida, R., Barbosa, J., \& Compte, A. (2015). Neural circuit basis of visuo-spatial

15 working memory precision: a computational and behavioral study. Journal of

16 Neurophysiology, 114(3), 1806-1818. http://doi.org/10.1152/jn.00362.2015

17 Barak, O., Sussillo, D., Romo, R., Tsodyks, M., \& Abbott, L. F. (2013). From fixed points

18 to chaos: Three models of delayed discrimination. Progress in Neurobiology, 103,

19 214-222. http://doi.org/10.1016/j.pneurobio.2013.02.002

20 Bays, P. M., \& Husain, M. (2008). Dynamic shifts of limited working memory

21 resources in human vision. Science, 321(5890), 851-854.

22 http://doi.org/10.1126/science.1158023

23 Bays, P. M., Catalao, R. F. G., \& Husain, M. (2009). The precision of visual working 
1 memory is set by allocation of a shared resource. Journal of Vision, 9(10), 7.1-11.

$2 \quad$ http://doi.org/10.1167/9.10.7

3 Ben-Yishai, R., Bar-Or, R. L., \& Sompolinsky, H. (1995). Theory of orientation tuning

$4 \quad$ in visual cortex. Proceedings of the National Academy of Sciences of the United

$5 \quad$ States of America, 92(9), 3844-3848.

6 Brady, T. F., \& Alvarez, G. A. (2011). Hierarchical Encoding in Visual Working

7 Memory: Ensemble Statistics Bias Memory for Individual Items. Psychological

8 Science, 22(3), 384-392. http://doi.org/10.1177/0956797610397956

9 Brady, T. F., \& Alvarez, G. A. (2015). Contextual effects in visual working memory

10 reveal hierarchically structured memory representations. Journal of Vision,

11 15(15), 6. http://doi.org/10.1167/15.15.6

12 Brady, T. F., \& Tenenbaum, J. B. (2013). A probabilistic model of visual working

13 memory: Incorporating higher order regularities into working memory capacity

14 estimates. Psychological Review, 120(1), 85-109.

15 http://doi.org/10.1037/a0030779

16 Brady, T. F., Konkle, T., \& Alvarez, G. A. (2009). Compression in visual working

17 memory: Using statistical regularities to form more efficient memory

18 representations. Journal of Experimental Psychology: General, 138(4), 487-502.

19 http://doi.org/10.1037/a0016797

20 Chatham, C. H., Frank, M. J., \& Badre, D. (2014). Corticostriatal Output Gatingduring

21 Selection from Working Memory. Neuron, 81(4), 930-942.

22 http://doi.org/10.1016/j.neuron.2014.01.002

23 Chen, Z., \& Cowan, N. (2005). Chunk Limits and Length Limits in Immediate Recall: A 
1 Reconciliation. Journal of Experimental Psychology. Learning, Memory, and Cognition, 31(6), 1235-1249. http://doi.org/10.1037/0278-7393.31.6.1235

3 Cohen, M. A., Rhee, J. Y., \& Alvarez, G. A. (2016). Limits on perceptual encoding can

4 be predicted from known receptive field properties of human visual cortex.

5 Journal of Experimental Psychology: Human Perception and Performance, 42(1), 67-77. http://doi.org/10.1037/xhp0000108

7 Collins, A. G. E., \& Frank, M. J. (2013). Cognitive control over learning: creating,

8 clustering, and generalizing task-set structure. Psychological Review, 120(1),

9 190-229. http://doi.org/10.1037/a0030852

10 Cowan, N. (2001). The magical number 4 in short-term memory: a reconsideration

11 of mental storage capacity. Behavioral and Brain Sciences, 24(1), 87-114-

12 discussion 114-85.

13 Cowan, N., \& Rouder, J. N. (2009). Comment on "Dynamic shifts of limited working

14 memory resources in human vision". Science, 323(5916), 877-author reply 877.

15 http://doi.org/10.1126/science.1166478

16 Curtis, C. E., \& D'Esposito, M. (2003). Persistent activity in the prefrontal cortex

17 during working memory. Trends in Cognitive Sciences, 7(9), 415-423.

18 http://doi.org/10.1016/S1364-6613(03)00197-9

19 Donkin, Chris, Tran, S. C., \& Nosofsky, R. (2013a). Landscaping analyses of the ROC

20 predictions of discrete-slots and signal-detection models of visual working

21 memory. Attention, Perception \& Psychophysics. http://doi.org/10.3758/s13414-

$22 \quad 013-0561-7$

23 Donkin, Christopher, Nosofsky, R. M., Gold, J. M., \& Shiffrin, R. M. (2013b). Discrete- 
1 slots models of visual working-memory response times. Psychological Review, 120(4), 873-902. http://doi.org/10.1037/a0034247

3 Durstewitz, D., \& Seamans, J. K. (2002). The computational role of dopamine D1

4 receptors in working memory. Neural Networks : the Official Journal of the

5 International Neural Network Society, 15(4-6), 561-572.

6 Ester, E. F., Sprague, T. C., \& Serences, J. T. (2015). Parietal and Frontal Cortex

$7 \quad$ Encode Stimulus-Specific Mnemonic Representations during Visual Working

8 Memory. Neuron, 87(4), 893-905. http://doi.org/10.1016/j.neuron.2015.07.013

9 Fallon, S. J., Zokaei, N., \& Husain, M. (2016). Causes and consequences of limitations

10 in visual working memory. Annals of the New York Academy of Sciences, n/a-n/a.

11 http://doi.org/10.1111/nyas.12992

12 Felsen, G., Touryan, J., \& Dan, Y. (2005). Contextual modulation of orientation tuning

13 contributes to efficient processing of natural stimuli. Network (Bristol, England), $14 \quad 16(2-3), 139-149$.

15 Fougnie, D., Cormiea, S. M., Kanabar, A., \& Alvarez, G. A. (2016). Strategic Trade-Offs

16 Between Quantity and Quality in Working Memory. Journal of Experimental

17 Psychology: Human Perception and Performance.

18 http://doi.org/10.1037/xhp0000211

19 Fougnie, D., Suchow, J. W., \& Alvarez, G. A. (2012). Variability in the quality of visual

20 working memory. Nature Communications, 3, 1229.

21 http://doi.org/10.1038/ncomms2237

22 Franconeri, S. L., Alvarez, G. A., \& Cavanagh, P. (2013). Flexible cognitive

23 resources:competitive content maps for attentionand memory. Trends in 
Cognitive Sciences, 17(3), 134-141. http://doi.org/10.1016/j.tics.2013.01.010

Frank, M. J., \& Badre, D. (2012). Mechanisms of Hierarchical Reinforcement Learning 509-526. http://doi.org/10.1093/cercor/bhr114

5 Freeman, E., Driver, J., Sagi, D., \& Zhaoping, L. (2003). Top-Down Modulation of Lateral Interactions in Early Vision. Current Biology, 13(11), 985-989. http://doi.org/10.1016/S0960-9822(03)00333-6

11 Fukuda, K., Vogel, E., Mayr, U., \& Awh, E. (2010). Quantity, not quality: the

12 relationship between fluid intelligence and working memory capacity.

13 Psychonomic Bulletin \& Review, 17(5), 673-679.

14 http://doi.org/10.3758/17.5.673

15 Funahashi, S., Bruce, C. J., \& Goldman-Rakic, P. S. (1989). Mnemonic coding of visual

16 space in the monkey's dorsolateral prefrontal cortex. Journal of Neurophysiology, $17 \quad 61(2), 331-349$.

18 Furman, M., \& Wang, X.-J. (2008). Similarity effect and optimal control of multiple-

19 choice decision making. Neuron, 60(6), 1153-1168.

20 http://doi.org/10.1016/j.neuron.2008.12.003

21 Fuster, J. M., \& Alexander, G. E. (1971). Neuron activity related to short-term 22 memory. Science.

23 Fuster, J. M., \& Jervey, J. P. (1981). Inferotemporal neurons distinguish and retain 
1 behaviorally relevant features of visual stimuli. Science, 212(4497), 952-955.

2 Gold, James M, Hahn, B., Zhang, W. W., Robinson, B. M., Kappenman, E. S., Beck, V. M.,

3 \& Luck, S. J. (2010). Reduced capacity but spared precision and maintenance of

$4 \quad$ working memory representations in schizophrenia. Archives of General

5 Psychiatry, 67(6), 570-577. http://doi.org/10.1001/archgenpsychiatry.2010.65

6 Goldman-Rakic, P. S. (1995). Cellular basis of working memory. Neuron, 14(3), 477-

$7 \quad 485$.

8 Gottlieb, J. (2004). Simultaneous Representation of Saccade Targets and Visual

9 Onsets in Monkey Lateral Intraparietal Area. Cerebral Cortex, 15(8), 1198-1206.

10 http://doi.org/10.1093/cercor/bhi002

11 Hazy, T. E., Frank, M. J., \& O’Reilly, R. C. (2006). Banishing the homunculus: Making

12 working memory work. Neuroscience, 139(1), 105-118.

13 http://doi.org/10.1016/j.neuroscience.2005.04.067

14 Hubel, D. H., \& Wiesel, T. N. (1959). Receptive fields of single neurones in the cat's

15 striate cortex. The Journal of Physiology, 148, 574-591.

16 http://doi.org/10.1113/jphysiol.2009.174151

17 Hubel, D. H., \& Wiesel, T. N. (1965). Receptive fields and functional architecture in

18 two nonstriate visual areas (18 and 19) of the cat. Journal of Neurophysiology,

$1928,229-289$.

20 Johnson, J. S., Spencer, J. P., Luck, S. J., \& Schöner, G. (2009). A Dynamic Neural Field

21 Model of Visual Working Memory and Change Detection. Psychological Science,

22 20(5), 568-577. http://doi.org/10.1111/j.1467-9280.2009.02329.x

23 Kilpatrick, Z. P., Ermentrout, B., \& Doiron, B. (2013). Optimizing Working Memory 
1

3 Kiyonaga, A., \& Egner, T. (2016). Center-Surround Inhibition in Working Memory.

with Heterogeneity of Recurrent Cortical Excitation. Journal of Neuroscience, 33(48), 18999-19011. http://doi.org/10.1523/JNEUROSCI.1641-13.2013 Current Biology : CB, 26(1), 64-68. http://doi.org/10.1016/j.cub.2015.11.013

Kohonen, T. (1982). Self-organized formation of topologically correct feature maps. Biological Cybernetics.

Kriete, T., Noelle, D. C., Cohen, J. D., \& O'Reilly, R. C. (2013). Indirection and symbol-

like processing in the prefrontal cortex and basal ganglia. Proceedings of the

National Academy of Sciences, 110(41), 16390-16395.

http://doi.org/10.1073/pnas.1303547110

Lara, A. H., \& Wallis, J. D. (2014). Executive control processes underlying multi-item working memory. Nature Publishing Group, 17(6), 876-883.

$$
\text { http://doi.org/10.1038/nn.3702 }
$$

Lara, A. H., \& Wallis, J. D. (2015). The Role of Prefrontal Cortex in Working Memory:

$$
\text { A Mini Review. Frontiers in Systems Neuroscience, 9, } 173 .
$$$$
\text { http://doi.org/10.3389/fnsys.2015.00173 }
$$

Lew, T. F., \& Vul, E. (2015). Ensemble clustering in visual working memory biases location memories and reduces the Weber noise of relative positions. Journal of

$$
\text { Vision, 15(4), 10. http://doi.org/10.1167/15.4.10 }
$$

Lowe, M. X., Stevenson, R. A., Wilson, K. E., Ouslis, N. E., Barense, M. D., Cant, J. S., \&

$$
\text { Ferber, S. (2016). Sensory processing patterns predict the integration of }
$$
information held in visual working memory. Journal of Experimental Psychology: 
http://doi.org/10.1037/xhp0000131

2 Luck, S. J., \& Vogel, E. K. (2013). Visual working memory capacity: from

3 psychophysics and neurobiology to individual differences. Trends in Cognitive

$4 \quad$ Sciences, 17(8), 391-400. http://doi.org/10.1016/j.tics.2013.06.006

5 Luria, R., \& Vogel, E. K. (2014). Come Together, Right Now: Dynamic Overwriting of an Object's History through Common Fate. Journal of Cognitive Neuroscience, 111. http://doi.org/10.1038/nature 02447

Ma, W. J., Husain, M., \& Bays, P. M. (2014). Changing concepts of working memory.

$9 \quad$ Nature Neuroscience, 17(3), 347-356. http://doi.org/10.1038/nn.3655

10 Matthey, L., Bays, P. M., \& Dayan, P. (2015). A Probabilistic Palimpsest Model of

11 Visual Short-term Memory. PLoS Computational Biology, 11(1), e1004003.

12 http://doi.org/10.1371/journal.pcbi.1004003.s001

13 Miller, E. K., \& Cohen, J. D. (2001). An integrative theory of prefrontal cortex

14 function. Annual Review of Neuroscience, 24, 167-202.

15 http://doi.org/10.1146/annurev.neuro.24.1.167

16 Miller, G. A. (1956). The magical number seven, plus or minus two: Some limits on

17 our capacity for processing information. Psychological Review.

18 Murray, J. D., Anticevic, A., Gancsos, M., Ichinose, M., Corlett, P. R., Krystal, J. H., \&

19 Wang, X. J. (2014). Linking Microcircuit Dysfunction to Cognitive Impairment:

20 Effects of Disinhibition Associated with Schizophrenia in a Cortical Working

21 Memory Model. Cerebral Cortex, 24(4), 859-872.

22 http://doi.org/10.1093/cercor/bhs370

23 O'Reilly, R. C., \& Frank, M. J. (2006). Making working memory work: a computational 
1

3 Oberauer, K., \& Lin, H.-Y. (2017). An interference model of visual working memory.

$4 \quad$ Psychological Review, 124(1), 21-59. http://doi.org/10.1037/rev0000044

5 Orhan, A. E., \& Jacobs, R. A. (2013). A probabilistic clustering theory of the

model of learning in the prefrontal cortex and basal ganglia. Neural Computation, 18(2), 283-328. http://doi.org/10.1162/089976606775093909 organization of visual short-term memory. Psychological Review, 120(2), 297-

328. http://doi.org/10.1037/a0031541

Orhan, A. E., \& Jacobs, R. A. (2014). Are performance limitations in visual short-term

memory tasks due to capacity limitations or model mismatch? arXiv.org.

Pratte, M. S., Park, Y. E., Rademaker, R. L., \& Tong, F. (2017). Accounting for stimulusspecific variation in precision reveals a discrete capacity limit in visual working memory. Journal of Experimental Psychology: Human Perception and

Performance, 43(1), 6-17. http://doi.org/10.1037/xhp0000302

Rademaker, R. L., Tredway, C. H., \& Tong, F. (2012). Introspective judgments predict the precision and likelihood of successful maintenance of visual working memory. Journal of Vision, 12(13), 21. http://doi.org/10.1167/12.13.21

Rouder, J. N., Morey, R. D., Cowan, N., Zwilling, C. E., Morey, C. C., \& Pratte, M. S. (2008). An assessment of fixed-capacity models of visual working memory.

Proceedings of the National Academy of Sciences, 105(16), 5975-5979.

http://doi.org/10.1073/pnas.0711295105

Sims, C. R., Jacobs, R. A., \& Knill, D. C. (2012). An ideal observer analysis of visual

22 working memory. Psychological Review, 119(4), 807-830.

23 http://doi.org/10.1037/a0029856 
1 Somers, D. C., Nelson, S. B., \& Sur, M. (1995). An emergent model of orientation

2 selectivity in cat visual cortical simple cells. The Journal of Neuroscience : the

3 Official Journal of the Society for Neuroscience, 15(8), 5448-5465.

4 Stephan, K. E., Penny, W. D., Daunizeau, J., Moran, R. J., \& Friston, K. J. (2009).

5 Bayesian model selection for group studies. NeuroImage, 46(4), 1004-1017.

6 http://doi.org/10.1016/j.neuroimage.2009.03.025

7 Störmer, V. S., \& Alvarez, G. A. (2014). Feature-Based Attention Elicits Surround

8 Suppression in Feature Space. Current Biology, 24(17), 1985-1988.

9 http://doi.org/10.1016/j.cub.2014.07.030

10 Swan, G., \& Wyble, B. (2014). The binding pool: A model of shared neural resources

11 for distinct items in visual working memory. Attention, Perception \&

12 Psychophysics, 76(7), 2136-2157. http://doi.org/10.3758/s13414-014-0633-3

13 Swan, G., Collins, J., \& Wyble, B. (2016). Memory for a single object has differently

14 variable precisions for relevant and irrelevant features. Journal of Vision, 16(3),

15 32. http://doi.org/10.1167/16.3.32

16 van den Berg, R., Awh, E., \& Ma, W. J. (2014). Factorial comparison of working

17 memory models. Psychological Review, 121(1), 124-149.

18 http://doi.org/10.1037/a0035234

19 van den Berg, R., Shin, H., Chou, W.-C., George, R., \& Ma, W. J. (2012). Variability in

20 encoding precision accounts for visual short-term memory limitations.

21 Proceedings of the National Academy of Sciences, 109(22), 8780-8785.

22 http://doi.org/10.1073/pnas.1117465109

23 Vogel, E. K., McCollough, A. W., \& Machizawa, M. G. (2005). Neural measures reveal 
1 individual differences in controlling access to working memory. Nature, 438(7067), 500-503. http://doi.org/10.1038/nature04171

3 Voytek, B., \& Knight, R. T. (2010). Prefrontal cortex and basal ganglia contributions

4 to visual working memory. Proceedings of the National Academy of Sciences,

5 107(42), 18167-18172. http://doi.org/10.1073/pnas.1007277107

6 Wang, X. J. (1999). Synaptic basis of cortical persistent activity: the importance of

$7 \quad$ NMDA receptors to working memory. Journal of Neuroscience, 19(21), 9587-

89603.

9 Warden, M. R., \& Miller, E. K. (2007). The Representation of Multiple Objects in

10 Prefrontal Neuronal Delay Activity. Cerebral Cortex, 17(suppl 1), i41-i50.

11 http://doi.org/10.1093/cercor/bhm070

12 Wei, Z., Wang, X.-J., \& Wang, D.-H. (2012). From distributed resources to limited

13 slots in multiple-item working memory: a spiking network model with

14 normalization. Journal of Neuroscience, 32(33), 11228-11240.

15 http://doi.org/10.1523/JNEUROSCI.0735-12.2012

16 Wilken, P., \& Ma, W. J. (2004). A detection theory account of change detection.

17 Journal of Vision, 4(12), 11-11. http://doi.org/10.1167/4.12.11

18 Xing, J., \& Heeger, D. J. (2001). Measurement and modeling of center-surround

19 suppression and enhancement. Vision Research, 41(5), 571-583.

20 Zhang, W., \& Luck, S. J. (2008). Discrete fixed-resolution representations in visual

21 working memory. Nature, 453(7192), 233-235.

22 http://doi.org/10.1038/nature06860

23 Zhang, W., \& Luck, S. J. (2009). Sudden death and gradual decay in visual working 
bioRxiv preprint doi: https://doi.org/10.1101/098939; this version posted November 2, 2017. The copyright holder for this preprint (which was not certified by peer review) is the author/funder, who has granted bioRxiv a license to display the preprint in perpetuity. It is made available under aCC-BY-NC-ND 4.0 International license.

1 memory. Psychological Science, 20(4), 423-428. http://doi.org/10.1111/j.1467-

$2 \quad 9280.2009 .02322 . x$

3 Zhang, W., \& Luck, S. J. (2011). The number and quality of representations in

$4 \quad$ working memory. Psychological Science, 22(11), 1434-1441.

$5 \quad$ http://doi.org/10.1177/0956797611417006

6

7

8

9

10

11

12

13

14

15

16

17

18

19

20

21

22

23 

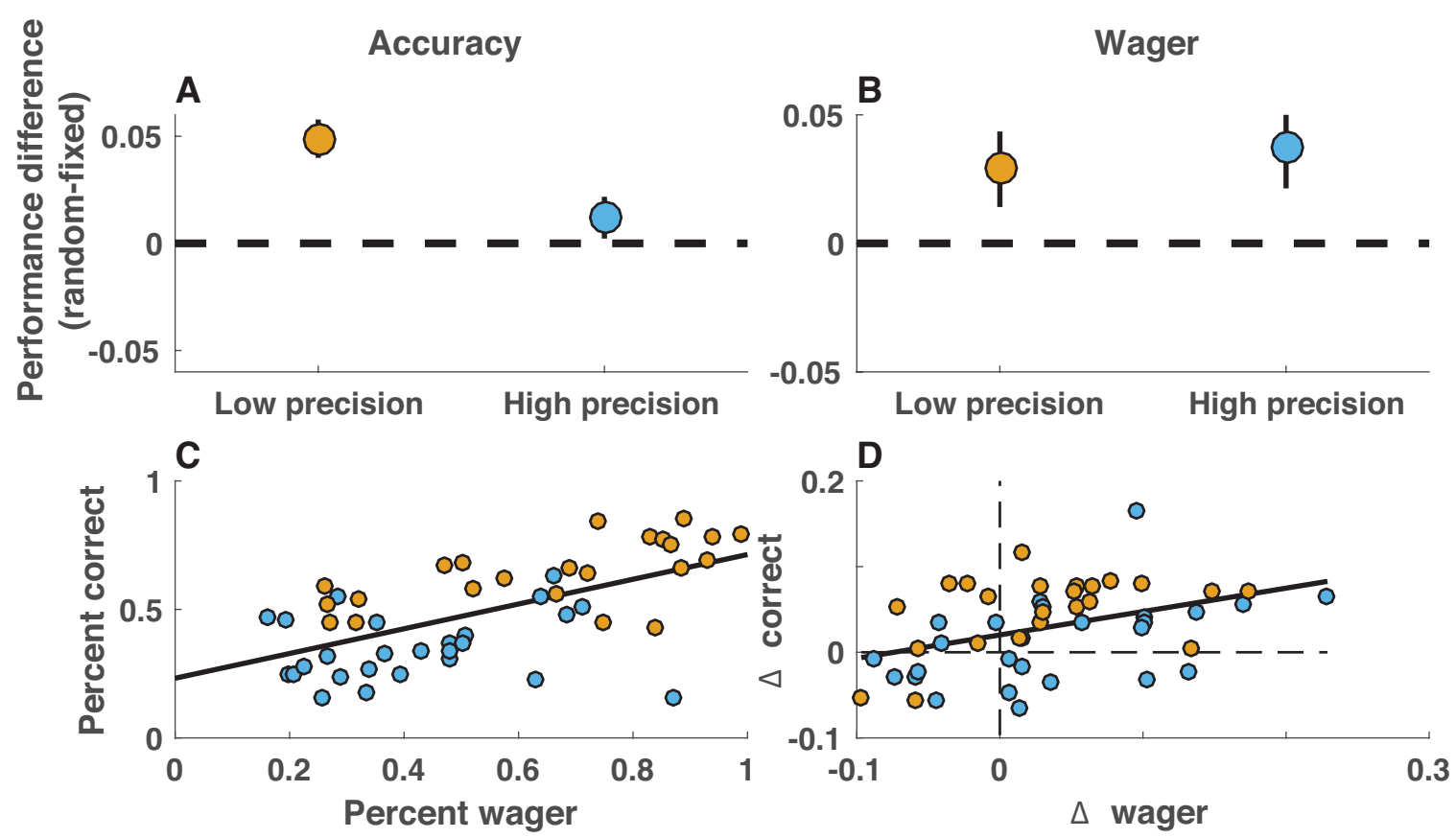

Figure S1: Uniformly spaced stimulus configurations degrade task performance and confidence in human subjects. Subject performance was assessed in terms of accuracy (percent of trials eliciting positive feedback) and confidence (percent of trials eliciting high post-decision wagers) separately according to precision condition (23 subjects were required to achieve an error of less than $\pi / 3$ to elicit positive feedback [low precision], whereas 24 subjects were required to achieve an error of less than $\pi / 8$ to elicit positive feedback [high precision]). A) Subjects in the low precision condition were more accurate for random spacing, as opposed to fixed spacing, stimulus configurations (orange; $\mathrm{t}=5.6, \mathrm{p}<10 \mathrm{e}-4$ ), whereas subjects in the high precision condition attained similar overall performance in both configurations (blue; $t=1.5, p=0.15$ ). Points/lines indicate group mean/SEM. B) Subjects in both conditions indicated higher confidence for random-spacing, as opposed to fixed-spacing, stimulus configurations $(t=[2.3,2.0]$ and $p=[0.03,0.06]$ for high and low precision conditions, respectively). C) Subjects that were most accurate, as assessed online according

15 to a fixed error threshold, also tended to make higher post-decision wagers. Orange and blue points 16 indicate subjects in low and high precision conditions, respectively. D) Furthermore, the

17 improvement in accuracy from fixed-to random-spaced arrays was greater for subjects that showed the largest increase in confidence across the same conditions. 


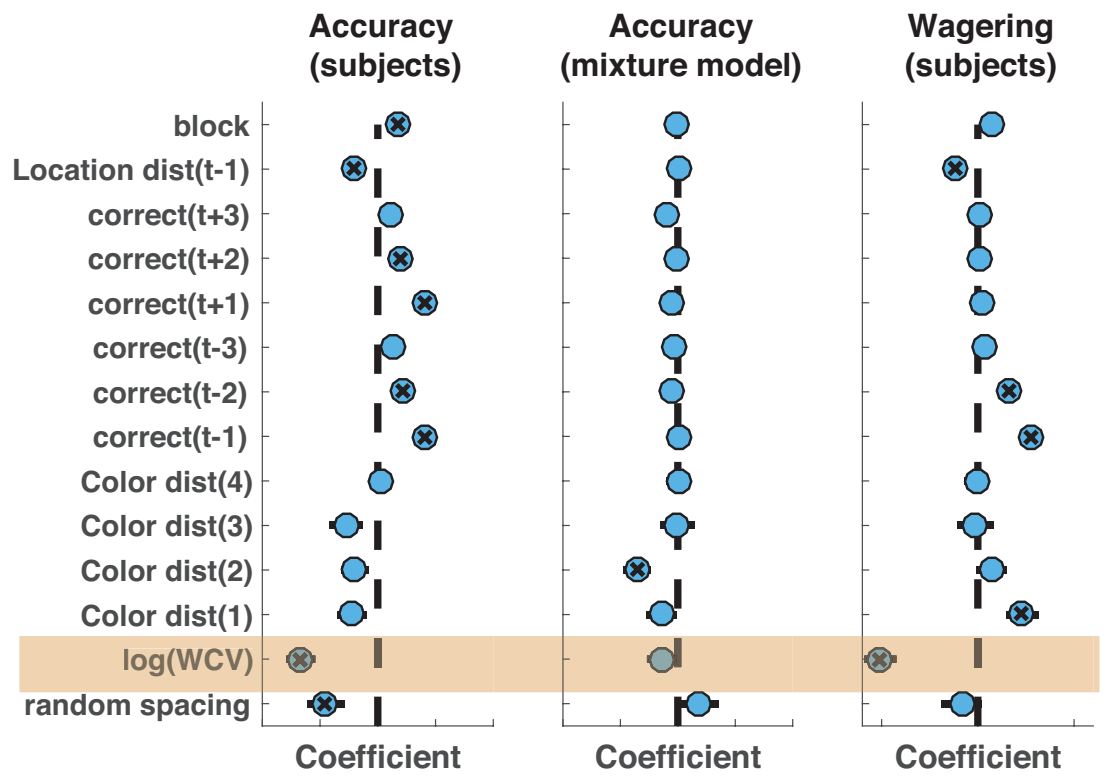



clustered. This effect is not present in accuracy data simulated from a mixture model that includes binding errors (center).

A

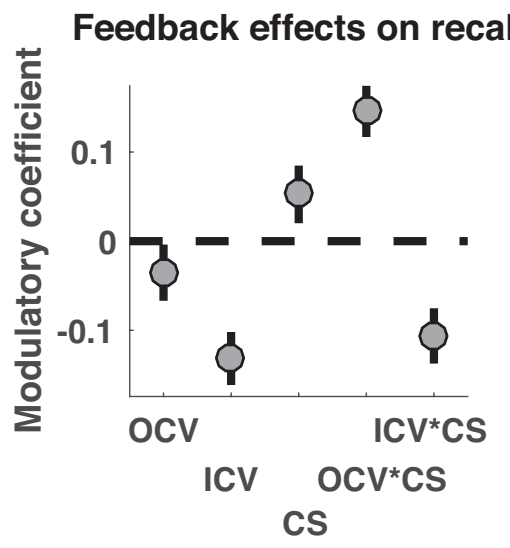

B

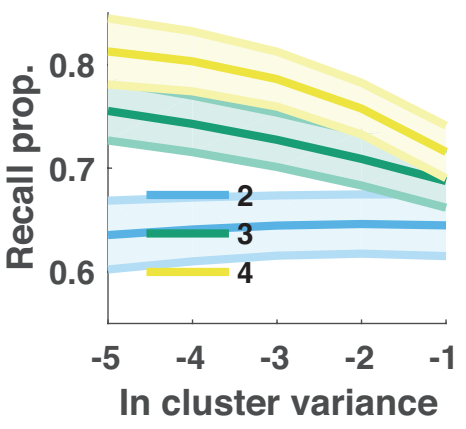

C

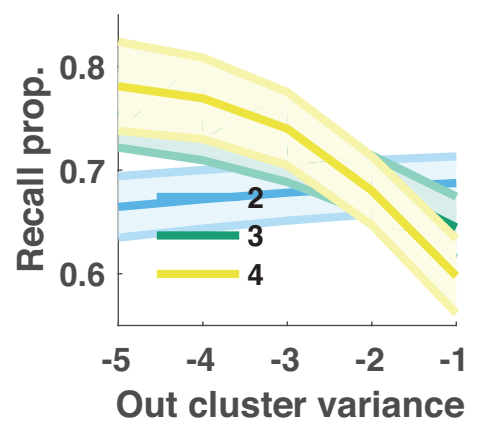

\section{Figure S3: Recall is affected by clustering of both probed and non-probed stimuli. To further} examine the source of the recall benefits, subject data were fit with a mixture model that considered reports to come from a mixture of processes including 1) a uniform "guess" distribution, 2) a "memory+binding" distribution centered on the color of the probed target, and 3) a "binding error" distribution including peaks at each non-probed target. Additional terms were included in the model to allow the recall probability to vary as a logistic function of various descriptive aspects of stimulus clustering that all factor into the within-cluster variance measurements reported in figure 5 . To do so, the color array from each trial was divided into two (minimal variance) clusters to compute 1) the variance of the cluster that did not contain the probed item [OCV], 2) the variance of the cluster that did contain the probed item [ICV], and the number of colors in the cluster that contained the probed target [CS]. A) Mean/SEM coefficients across subjects indicated that these three factors, along with their interactions, were systematically related to trial-to-trial fluctuations in subject recall rates. B\&C) The predicted recall rates from model fits are plotted as a function of ICV (B) and OCV (C) color coded according to the number of items in the relevant cluster (the cluster containing the probed item for B, and the cluster that did not contain the probed item for $\mathrm{C}$ ). Recall bonuses are evident for low values of both ICV and OCV, although these benefits scale with the number of colors contained in the relevant cluster. 


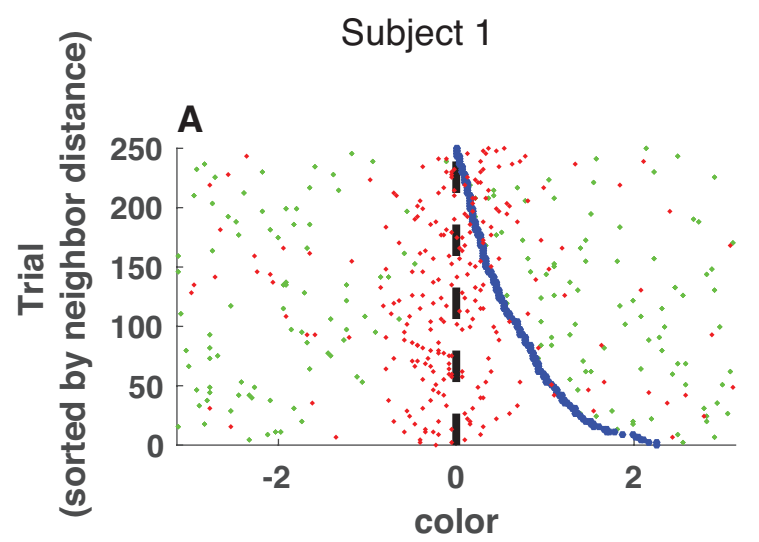

Subject 31
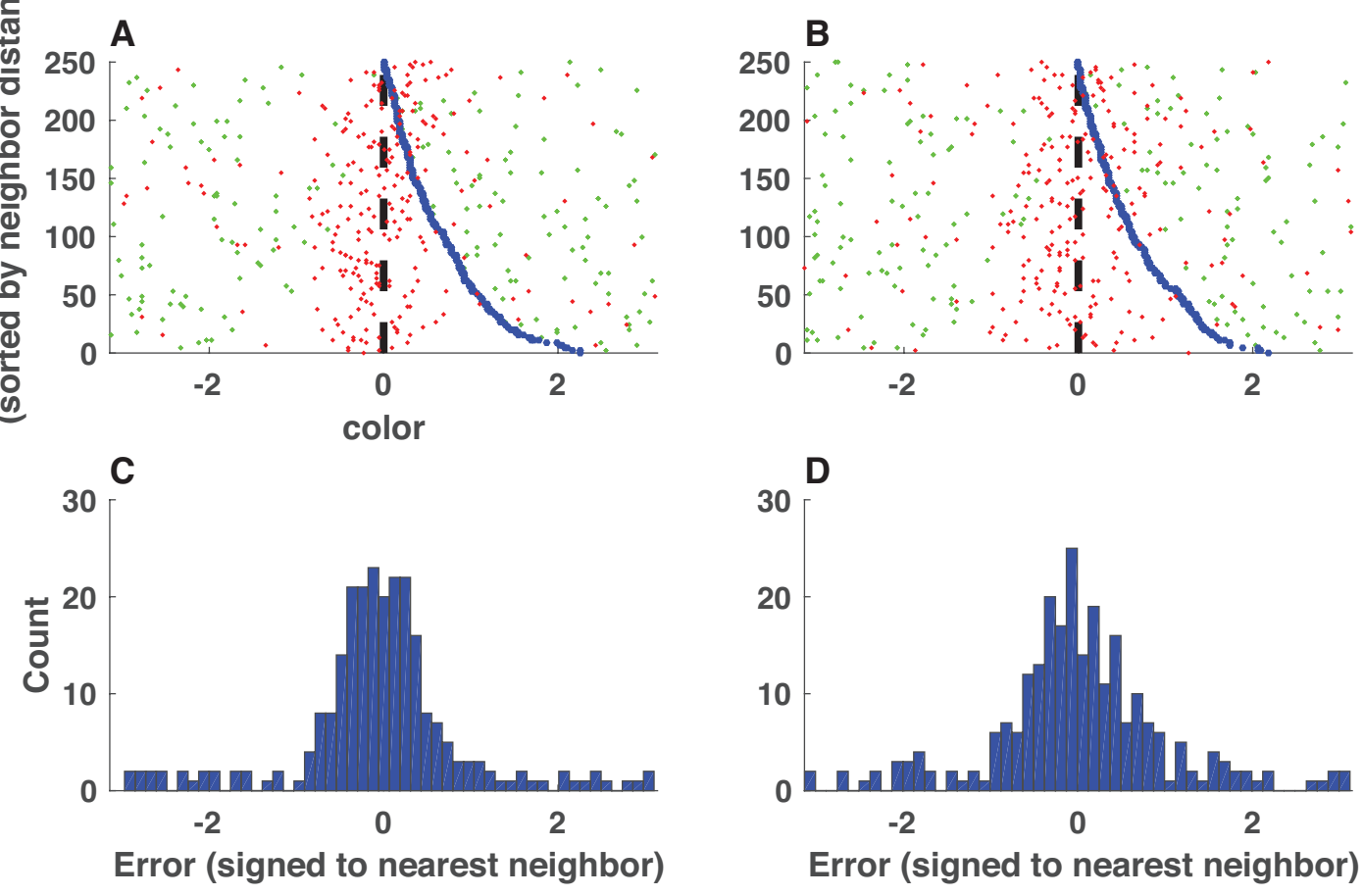

Figure S4: Sorting trials according to the nearest neighbor non-probed target color reveals structure in memory reports. A\&B: Signed error of memory reports (red points) for all trials completed by two sample subjects (left $=$ subject 1 , right $=$ subject 31 ). Trial errors are sorted by the distance from the probed target to the most similar color in the target array (nearest neighbor distance, NND) and transformed according to the direction of the nearest neighbor target (blue points). Green points reflect the positions of other colors in the target array, relative to the probed color and transformed as described above. Note the asymmetry in error distributions appears to change as a function of the nearest neighbor distance. C\&D: Error histograms for the same two example subjects, transformed as described above. Note that in some cases apparent structure in the sorted errors (A) is no longer visible after collapsing across nearest neighbor distances (C).

14 

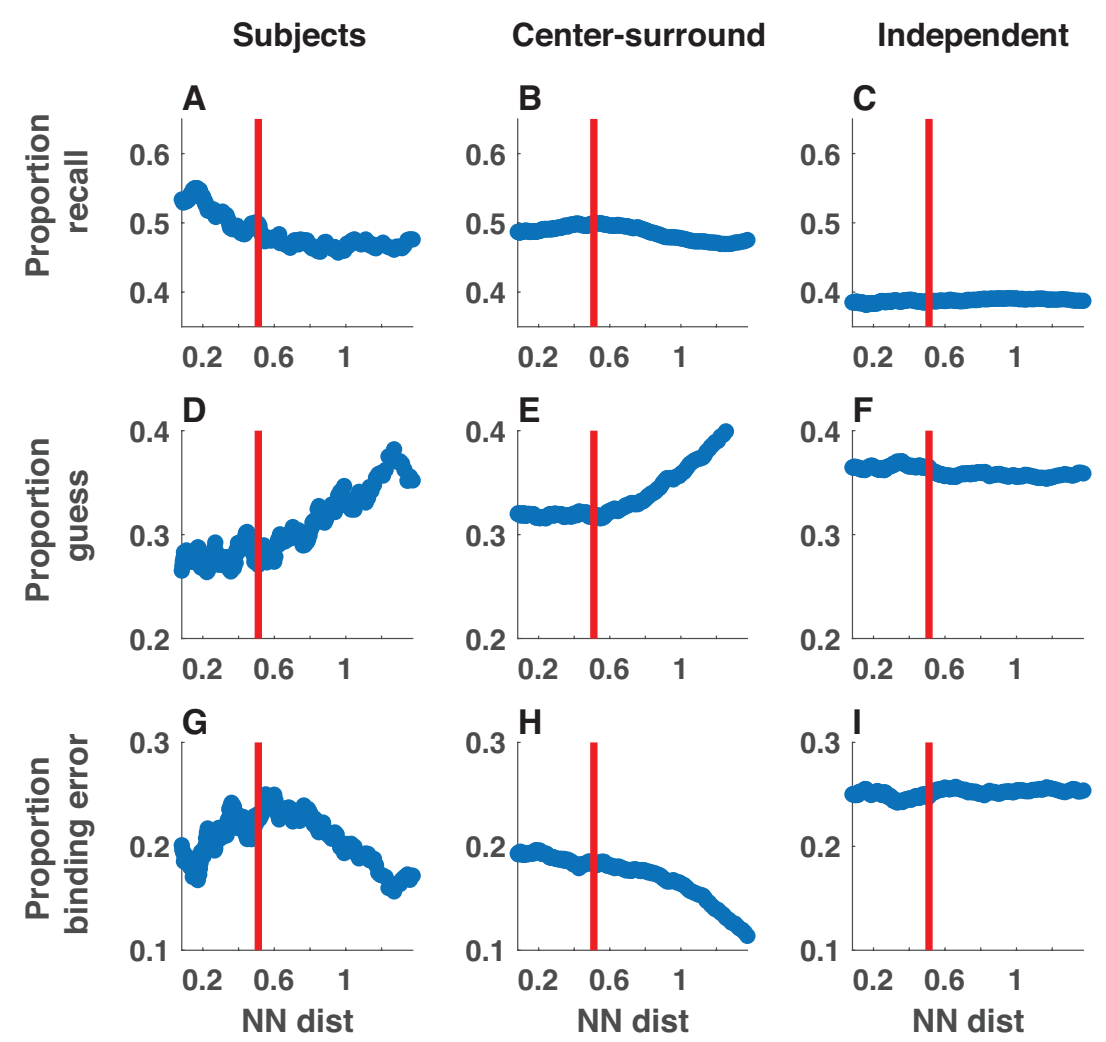

Figure S5: Neighboring stimulus features affect fits of mixture model. Subject (left) and simulated (center $=$ center-surround, right $=$ independent encoding) data were collapsed across all sessions and binned in sliding windows according to the absolute distance between the probed target color and the most similar non-probed target color (NN dist; abscissa). Data in each bin were fit with a mixture model that included free parameters to estimate the proportion of reports generated from 1) the von Mises "memory distribution" (A-C), 2) the uniform "guess distribution" (D-F), or 3)the mixture of von Mises "binding error distribution" (G-I). Parameter estimates for precision and bias terms are reported in the main text (figure 8). 